NBER WORKING PAPER SERIES

\title{
SHOULD HOSPITALS KEEP THEIR PATIENTS LONGER? THE ROLE OF INPATIENT CARE IN REDUCING POST-DISCHARGE MORTALITY
}

\author{
Ann P. Bartel \\ Carri W. Chan \\ Song-Hee (Hailey) Kim \\ Working Paper 20499 \\ http://www.nber.org/papers/w20499 \\ NATIONAL BUREAU OF ECONOMIC RESEARCH \\ 1050 Massachusetts Avenue \\ Cambridge, MA 02138 \\ September 2014
}

Previously circulated as "Should Hospitals Keep Their Patients Longer? The Role of Inpatient and Outpatient Care in Reducing Readmissions." The views expressed herein are those of the authors and do not necessarily reflect the views of the National Bureau of Economic Research.

NBER working papers are circulated for discussion and comment purposes. They have not been peerreviewed or been subject to the review by the NBER Board of Directors that accompanies official NBER publications.

(C) 2014 by Ann P. Bartel, Carri W. Chan, and Song-Hee (Hailey) Kim. All rights reserved. Short sections of text, not to exceed two paragraphs, may be quoted without explicit permission provided that full credit, including $(\subset$ notice, is given to the source. 
Should Hospitals Keep Their Patients Longer? The Role of Inpatient Care in Reducing PostDischarge Mortality

Ann P. Bartel, Carri W. Chan, and Song-Hee (Hailey) Kim

NBER Working Paper No. 20499

September 2014

JEL No. I10,I13,I18,L38

\begin{abstract}
The Centers for Medicare \& Medicaid Services (CMS) and the National Quality Forum have endorsed 30-day mortality rates as important indicators of hospital quality. Concerns have been raised, however, as to whether post-discharge mortality rates are reasonable measures of hospital quality as they consider the frequency of an event that occurs after a patient is discharged and no longer under the watch and care of the hospital. Using a large dataset comprised of all hospital encounters of every Medicare patient from 2000 to 2011 and an instrumental variables methodology to address the potential endogeneity bias in hospital length-of-stay, we find evidence that 30-day mortality rates are appropriate measures of hospital quality. For patients with diagnoses of Pneumonia or Acute Myocardial Infarction, an additional day in the hospital could decrease 30 -day mortality rates by up to $12.8 \%$. Moreover, we find that, from a social planner's perspective, the gains achieved in reducing mortality rates far exceed the cost of keeping these patients in the hospital for an additional day.
\end{abstract}

Ann P. Bartel

Graduate School of Business

Columbia University

3022 Broadway, 623 Uris Hall

New York, NY 10027

and NBER

apb2@columbia.edu

Carri W. Chan

Columbia University

Graduate School of Business

3022 Broadway, 410 Uris Hall

New York, NY 10027

cc3179@columbia.edu
Song-Hee (Hailey) Kim

University of Southern California

Marshall School of Business

Bridge Hall 307A

3670 Trousdale Parkway

Los Angeles, CA 90089

songheek@marshall.usc.edu 


\title{
Should Hospitals Keep Their Patients Longer? The Role of Inpatient Care in Reducing Post-Discharge Mortality
}

\author{
Ann P. Bartel \\ Finance \& Economics, Columbia Business School; NBER, apb2@columbia.edu \\ Carri W. Chan \\ Decision, Risk, and Operations, Columbia Business School, cwchan@columbia.edu \\ Song-Hee Kim \\ Data Sciences and Operations, Marshall School of Business, University of Southern California, songheek@marshall.usc.edu \\ This version: March 22, 2016
}

Key words: Healthcare, Medicare, Econometric Analysis, Length-of-stay, Mortality, Hospital quality

\section{Introduction}

The National Quality Forum (NQF), a non-profit organization that conducts research on hospital quality measures, and the Centers for Medicare and Medicaid Services (CMS) provide various measures of hospital quality which are accessible to the public. Both of these organizations publish their measures of hospital quality for two main purposes: (1) to provide information to potential patients regarding the quality of care at different hospitals and (2) to encourage hospitals to improve their quality of care. When CMS first launched the Hospital Compare website (www.medicare.gov/hospitalcompare/) in 2005, only process-ofcare measures, such as the percentage of Pneumonia patients given the most appropriate initial antibiotic(s), were reported. By 2008, the Centers for Medicare \& Medicaid Services (CMS) began reporting outcome measures such as 30-day mortality for acute myocardial infarction (AMI), heart failure (HF), and pneumonia (PNE) on the website (Centers for Medicare \& Medicaid Services 2015). CMS's perspective that 30-day mortality rates are an important measure of quality is made clear under the Hospital Value Based Purchasing plan, which began in Fiscal Year 2013. Under this plan, hospital reimbursements are adjusted based on a Total Performance Score, which includes 30-day mortality rates for AMI, HF, and PNE (Centers for Medicare \& Medicaid Services 2016). NQF also endorses 30-day mortality as a hospital quality measure because "it allows for a broad view of quality of care that encompasses more than what can be captured by individual process-of-care measures" (National Quality Forum 2009). 
Although both CMS and NQF report 30-day mortality rates as a measure of hospital quality, there are conflicting views as to whether the reported 30-day mortality measure is a fair measure of quality. Indeed, there is an "ongoing debate about Hospital Compare, whose measures, critics say, do not necessarily reflect quality of care provided at hospitals" (Fleming 2012). One argument is that 30-day mortality, defined as whether a patient dies within 30 days of hospital discharge, may be more influenced by what happens after hospital discharge. Some people have raised questions as to whether it is fair to attribute post-discharge outcomes to hospitals whose focus is on inpatient care delivery. With this in mind, in-hospital mortality (rather than post-discharge mortality) is often considered as a measure of quality (e.g. Clark and Huckman (2012), KC et al. (2013), Kuntz et al. (2015)). On the other hand, hospitals play a large role in what happens to patients once they leave the hospital in that they typically arrange for follow-up appointments as well as communicate with follow-up care providers such as the primary care and specialist physicians. In this respect, "[i]t's reasonable to say that hospitals have some responsibility for what happens when the patient leaves" (Clark 2012).

Our study contributes to this debate by exploring whether there are factors related to inpatient care which can impact the post-discharge outcome of 30-day mortality. Studies of process-of-care measures and their impact on mortality have led to mixed conclusions. For example, Jha et al. (2007) found that better process-of-care measures reported on Hospital Compare are related to lower risk-adjusted inpatient mortality, while Ryan et al. (2009) found that when controlling for unobserved heterogeneity, the processof-care performance measures are not associated with 30-day mortality. The authors conclude that "this suggests that the relationship between hospital-level process-of-care performance and [30-day] mortality is not causal."

In contrast, our paper considers the impact of increasing a patient's hospital length-of-stay (LOS), with the idea that an extra day in the hospital may provide benefits such as allowing a patient to reach a higher level of stability as well as providing more time for patients to be educated about expectations with respect to their post-discharge behavior, thereby resulting in a reduction in the risk of mortality. There have been a number of articles that have studied the relationship between LOS and mortality. Focusing on Medicare fee-for-service patients hospitalized for HF during the time period 1993-2006, Bueno et al. (2010) documented a decrease in hospital LOS and found that mortality rates had either stayed the same or increased, but they were unable to show a causal relationship between these trends. Chan et al. (2012) found no association between shorter ICU LOS and in-hospital mortality. Other papers found that an increase in LOS was associated with an increase in the risk of post-discharge mortality (e.g. Williams et al. (2010), Nichols et al. (2014), Reynolds et al. (2015)). Looking at patients with similar ailments to those we study, Kaboli et al. (2012) examined Veterans Hospitals and found that reductions in LOS from 1997 to 2010 did not come at 
the expense of higher mortality. Clark (2012) argued that some hospitals may be 'cherry-picking' healthier patients who have shorter LOS and lower mortality rates or they may even be discharging/transferring patients with poor prognoses, so that "they look better when their death rates are compared with hospitals that keep patients longer." While these papers considered the relationship between LOS and mortality, none of them conducted a rigorous study regarding the causal effect of shorter LOS increasing post-discharge mortality.

Utilizing an instrumental variables methodology, we measure the impact of an increase in hospital LOS on the mortality rates for all Medicare patients hospitalized for AMI, PNE, or HF. Our dataset is from the Centers for Medicare \& Medicaid Services (CMS) and consists of all Medicare in-hospital patient visits between 2000 and 2011. Estimating the impact of LOS on the probability of post-discharge mortality is complicated; it is not possible to perfectly measure a patient's severity level and unobservable severity factors might be positively correlated with both LOS and mortality risk. To address the possible endogeneity in LOS, we use an instrument that is based on a patient's admission day-of-week. In our data, we find that the residuals from a LOS equation for patients admitted early in the week (e.g. on Monday) are negative, suggesting that they are 'prematurely' discharged. The average LOS for all three patient diagnoses is approximately 5 days, which implies that patients admitted early in the week would be ready for discharge on the weekend. However, because hospitals prefer to discharge patients before the weekend (see Varnava et al. (2002) and Wong et al. (2009)), these patients end up with a shorter than normal LOS. This variation in LOS based on admission day-of-week helps us capture the impact of shorter LOS on increased mortality risk. Our analysis also considers the fact that there may be unobservable patient severity factors (e.g. only really sick patients who cannot delay their treatment will go to hospitals on the weekend) and/or operational factors (e.g. availability of resources or specialists) which vary by admission day-of-week that influence mortality risk. To circumvent the concerns that patient severity might differ by day-of-week, our main analysis focuses on non-elective patients whose admission diagnosis is AMI or PNE because these diagnoses can be considered to be "non-deferrable" (Card et al. 2009), i.e. admissions are equally likely on the weekdays and weekend. Patients with HF, a chronic condition, do not satisfy the non-deferrability criterion and our estimates of the impact of LOS on their mortality risk may not be valid. To mitigate concerns about variation in hospital resource availability by day-of-week, we make a design choice to exclude weekend admits.

We present compelling evidence that keeping AMI and PNE patients in the hospital for one more day could decrease mortality rates by up to $12.8 \%$. Using hospital cost estimates and value of life estimates, we calculate that, for these conditions, the impact of keeping a patient one more day in the hospital is costeffective from a social welfare perspective. Moreover, we find that our results are very robust to a range of 
estimates of hospital costs and the value of life. One interpretation of our findings is that hospitals should consider moving to a 7-day discharge cycle, thereby reducing the likelihood of premature discharge due to the weekend.

In addition to 30-day mortality rates, Hospital Compare also reports 30-day readmission rates, a condition that has also been debated as a reasonable measure of quality (Jha 2013, Press et al. 2013). The prior literature has found mixed evidence as to whether shortened LOS increases the risk of readmission. Jaeker and Tucker (2015) found no association between hospital LOS and readmissions. On the other hand, Kc and Terwiesch (2012) found that for some cardiac surgery patients in Intensive Care Units (ICU), a reduction in ICU LOS resulted in an increase in ICU readmission risk, while Carey (2015) and Makowsky and Klein (2015) found a similar relationship for AMI and HF patients hospitalized in New York State and California, respectively. We briefly examine hospital readmissions and our results are consistent with this literature in that we find that LOS likely does not have a significant effect in reducing readmissions.

The remainder of the paper is structured as follows: Section 2 describes the dataset and the sample we use for our analyses. Section 3 describes our econometric model and explains why admission day-of-week is a valid instrument in our study setting. In Section 4 we present our main results, including a number of robustness checks, for AMI and PNE patients. Because HF is a chronic condition, we consider it separately in Section 5. Section 6 briefly examines readmissions. In Section 7, we discuss the policy implications of our findings from a social planner's perspective and also consider the implications of our findings for hospital administrators. We conclude in Section 8.

\section{Setting}

\subsection{Data}

We utilize data on all inpatient hospitalizations from 2000-2011 for Medicare Fee-For-Service (FFS) beneficiaries. Medicare FFS is the typical version of Medicare under which 70-80\% of beneficiaries are covered. These data are drawn from the 100\% sample in the Medicare Provider Analysis and Review (MedPAR) inpatient file ${ }^{1}$.

Our observations are at the patient-visit level. For each hospitalization, we have the patient's demographic information including age, gender, race, coverage choice, and hospitalization characteristics including admission and discharge dates (which enable us to compute the patient's LOS and account for potential seasonal variations), the primary condition or other coexisting conditions identified by up to 10 International Classification of Diseases, Ninth Revision, Clinical Modification (ICD-9-CM) codes, the Medicare Severity adjusted Diagnosis Related Group (MS-DRG) classification (indicating the DRG to which the claims that

\footnotetext{
${ }^{1}$ See http://www.resdac.org/cms-data/files/medpar-rif for a description of this dataset.
} 
comprise the stay belong for payment purposes), hospital, and admission type (e.g., elective or emergency basis). We also generate a severity of illness measure, the Elixhauser index (Elixhauser et al. 1998), using the ICD-9-CM codes and the MS-DRG classification.

\subsection{Patient Outcomes}

Given the reporting requirements of Hospital Compare, the main patient outcome of this study is 30-day mortality, defined as death within 30 days of discharge from a hospitalization. Our goal is to understand the impact on post-discharge mortality of keeping a patient in the hospital one more day. In addition to 30-day mortality, we also consider 3-day and 7-day mortality since inpatient care may be more likely to impact short-term mortality risk. Finally, for completeness, we also include 60-day mortality. Note that in all four of the mortality models, we exclude patients who die during their hospitalization.

\subsection{Selection of Patient Samples}

We examine three distinct patient samples-patients with Acute Myocardial Infarction (AMI), Heart Failure (HF), or Pneumonia (PNE)-because these were the first three diagnoses for which mortality rates were reported on Hospital Compare ${ }^{2}$. We use the primary ICD-9 codes to identify patients with these ailments.

Appendix Tables A.1 through A.3 describe our sample selection process for AMI patients, HF patients, and PNE patients, respectively. We only consider hospital stays with admission and discharge that occur between January 1, 2000 and October 31, 2011. Because we study 60-day mortality, an event which occurs within 60 days of discharge, we exclude admissions and discharges that occur during November and December 2011 to avoid potential censoring of our outcome variables. Due to data fidelity concerns, we exclude visits with overlapping admissions (i.e., admissions that occur prior to discharge of the previous hospital stay). Following CMS (Grady et al. 2013), we focus on acute care stays. Stays that involve hospital transfers are excluded as it is difficult to control for what happens in two different hospitals and during the transfer time.

We exclude stays that are not paid under the current DRG code based prospective payment system (PPS) which Medicare switched to in 1983; after this significant payment change, patient care also began to change since payments were no longer based on the amount of time patients spent in the hospital, but rather based on the average cost to treat the particular DRG.

We then keep the patients with the specific conditions on which we are focusing: AMI, HF, or PNE. Following CMS (Grady et al. 2013), we exclude admissions within 30 days of a prior hospitalization's discharge. Since the hospitals with fewer than 25 visits for each corresponding condition do not have their

\footnotetext{
${ }^{2}$ Note that the mortality reporting requirements have since been expanded to include: chronic obstructive pulmonary disease (COPD), stroke, and Coronary Artery Bypass Graft (CABG). However, as the initial program focuses on AMI, HF, and PNE, we do as well.
} 
performance publicly reported, we exclude hospitals that have less than 25 visits for each condition. Patients who are discharged to destinations that provide inpatient related services are excluded. We only include patients 65 years and older, which is the primary indication for Medicare eligibility. Next, we exclude patients who died during their hospital stay, who left against medical advice, who do not have their race reported, and who do not reside in the U.S.

We focus on emergency and urgent (i.e., non-elective) patients to leverage the random variation in admission day-of-week to construct an instrumental variable (see Section 3.1 for details). Such an identification strategy is not possible for elective patients whose admissions are mostly scheduled. Finally, we exclude patients who are LOS outliers (greater than the 99th percentile value) and cost outliers (as identified in the MedPAR inpatient file).

\subsection{Summary Statistics}

Table 1 presents means and standard deviations for the three patient samples (AMI, HF and PNE). Table 1 shows that AMI patients have marginally higher short-term mortality rates than HF and PNE (2\% and $3 \%$ versus $1 \%$ and $2 \%$ for 3 -day and 7-day mortality, respectively), while the longer-term mortality rate for AMI patients ( $7 \%$ and $11 \%$ for 30-day and 60-day mortality) is comparable (or better) than HF ( $8 \%$ and $12 \%$ ) and PNE (7\% and 12\%). The average LOS for these patients ranges between 4.9 days for HF patients to 5.5 days for PNE patients. Moreover, there is quite a bit of variation in length of stay depending on the day of admission with an over 5\% increase between the shortest LOS for Sunday or Monday admits and the longest LOS for Friday admits; in Section 3, we discuss how this variation enables us to construct an instrument to deal with the bias attributable to unobservable patient severity characteristics.

In our analysis we will first consider the acute conditions, AMI and PNE, and then consider the chronic condition, HF, separately. Note that the sample sizes given in Table 1 may not be exactly equivalent to the sample sizes in our regressions in Sections 4 to 6. This is because 1) a few patients stayed in the hospital for less than 1 full day and, as will be explained below, our model uses logarithm of hospital LOS as a dependent variable, thereby requiring a LOS of at least 1 day; and 2) some samples are dropped because they are perfect predictors of the outcome of interest ${ }^{3}$.

\section{Econometric Model}

Our goal is to estimate the impact of hospital LOS on 3-, 7-, 30-, and 60-day mortality. The mortality measure of interest is denoted by $D^{(t)}$, where $t$ is the number of days following hospital discharge, e.g. $D^{(3)}$ corresponds to 3-day mortality. We start with the following equation:

$$
y_{i}^{*}=\beta X_{i}+\theta \log \left(L O S_{i}\right)+\xi M_{i}+\psi Y R_{i}+\eta H_{i}+\epsilon_{i}
$$

\footnotetext{
${ }^{3}$ For example, if all patients in hospital $i$ die within 30 days of discharge, then a hospital fixed effect for hospital $i$ would be a
} perfect predictor of mortality and all patients treated in hospital $i$ would be dropped from the mortality regression. 
Table 1 Summary statistics

\begin{tabular}{l|cc|c}
\hline & \multicolumn{2}{|c|}{ Acute Diagnoses } & Chronic Diagnosis \\
\cline { 2 - 4 } & AMI & PNE & HF \\
\hline Num. of obs. & 1600420 & 3686544 & 4047914 \\
Age & 78.4 & 79.6 & 80.0 \\
Elixhauser Score & $(8.3)$ & $(8.2)$ & $(8.1)$ \\
& 2.1 & 2.7 & 2.6 \\
Female & $(1.4)$ & $(1.4)$ & $(1.5)$ \\
Race - White & 0.50 & 0.55 & 0.58 \\
Race - Black & 0.89 & 0.88 & 0.83 \\
Race - Hispanic & 0.08 & 0.07 & 0.13 \\
Race - Other & 0.02 & 0.02 & 0.02 \\
Had surgical procedure(s) & 0.02 & 0.02 & 0.02 \\
& 0.71 & 0.27 & 0.34 \\
Average LOS (days) & & & \\
Sunday & 5.4 & 5.5 & 4.9 \\
Monday & $(3.4)$ & $(3.3)$ & $(3.1)$ \\
Tuesday & 5.4 & 5.3 & 4.7 \\
Wednesday & 5.2 & 5.4 & 4.8 \\
Thursday & 5.3 & 5.4 & 4.8 \\
Friday & 5.4 & 5.5 & 4.9 \\
Saturday & 5.5 & 5.6 & 5.1 \\
& 5.6 & 5.6 & 5.1 \\
\% Admitted & 5.5 & 5.4 & 4.9 \\
Sunday & & & \\
Monday & & & \\
Tuesday & 13.6 & 13.3 & 12.1 \\
Wednesday & 15.5 & 16.2 & 17.3 \\
Thursday & 14.5 & 14.8 & 15.5 \\
Friday & 14.3 & 14.2 & 14.6 \\
Saturday & 14.2 & 14.2 & 14.4 \\
& 14.4 & 14.5 & 14.5 \\
Death in 3 days & 13.5 & 12.9 & 11.6 \\
Death in 7 days & & & \\
Death in 30 days & 0.02 & 0.01 & 0.01 \\
Death in 60 days & 0.03 & 0.02 & 0.02 \\
\hline Nore Aves & 0.08 & 0.07 \\
& 0.12 & 0.12 \\
\hline
\end{tabular}

Note. Averages (standard deviation in parentheses for continuous variables) are reported.

$$
y_{i}=1_{\left\{y_{i}^{*}>0\right\}}
$$

where $y_{i}$ is the binary outcome of interest and can be equal to any of the four mortality measures, $D^{(t)}$. Thus, $y_{i}^{*}$ can be interpreted as the latent risk of death occurring in the specified time frame.

In equation (1), $X_{i}$ is a vector of patient characteristics: age, gender, race, Elixhauser co-morbidities ${ }^{4}$,

${ }^{4}$ Elixhauser et al. (1998) defines 30 comorbid conditions using the ICD-9-CM and MS-DRG codes. Equation (1) includes 30 
DRG code, and a dummy variable for having one or more surgical procedures (any minor/major diagnostic or therapeutic procedures $)^{5} . M_{i}, Y R_{i}$ and $H_{i}$ are all vectors: $M_{i}$ is the month of hospital admission; $Y R_{i}$ is the year of hospital admission; and, $H_{i}$ is the hospital in which patient $i$ is treated. Hence, we include month and year dummies as well as hospital fixed effects; the inclusion of the hospital fixed effects controls for the potential impact of unobservable attributes of the more than 3000 hospitals in our study. As is standard practice, we take the logarithm of the patient's LOS in order to account for the heavy tails in this distribution. We assume that the error term $\epsilon_{i}$ is a standard normal random variable to fit the Probit model.

While the Elixhauser co-morbid conditions have been widely used in previous research, these measures are not a perfect control for patient severity. Unobservable severity factors might be positively correlated with both LOS and the dependent variable in equation (1). Since sicker patients tend to stay longer in the hospital and are also more likely to die, we might draw an erroneous conclusion that longer LOS leads to higher mortality risks. In the following subsection, we describe our instrumental variable approach to address this possible endogeneity in $\log (L O S)$.

\subsection{Instrument for LOS}

A valid instrumental variable (IV) is correlated with the endogenous variable $(\log (L O S))$ and uncorrelated with the unobservable noise (Wooldridge 2010). We now propose an IV that is based on a patient's admission day-of-week and evaluate whether it satisfies these two properties. A number of studies have used the timing of admission by using time-of-day or day-of-week as an IV in other healthcare settings as the timing of admission has been shown to have an impact on the type of care patients receive (e.g. Ho et al. (2000), Hamilton et al. (2000), Ryan et al. (2005), Goyal et al. (2013), Baiocchi et al. (2014)).

Unobserved Severity by Admission Day-of-Week: For an IV to be valid, it must be uncorrelated with the unobservable noise; in our case, the admission day-of-week must be uncorrelated with the unobserved severity of the patient condition. We follow the approach used by Card et al. (2009) who study patients in California and define non-deferrable diagnoses as those for which admissions are equally likely on the weekend and weekdays. The rationale behind this approach is that patients will only go to the hospital on the weekend if it is truly necessary, i.e. their condition is 'non-deferrable'. As such, it is unlikely that there are unobservable severity measures correlated with admission day of week for patients with non-deferrable diagnoses-each day of the week is considered the same as any other day of the week. Specifically, for

dummy variables, one for each of the 30 conditions.

${ }^{5}$ While we do not have any data on the socio-economic status of the patients, we believe this is of minimal concern. Although we expect patients with lower socio-economic status to be more likely to die, we also expect them to be less likely to be prematurely discharged as hospitals are hesitant to send patients home without a solid support system to help manage their recovery. If there were a positive correlation between premature discharge and socio-economic status, this could result in us erroneously concluding that premature discharge increases the likelihood of mortality when the true effect may be due to socio-economic status. 
each ICD9 code, we calculate t-statistics to see if the proportion of patients admitted on the weekend is significantly different from $2 / 7$ of the total weekly admissions ${ }^{6}$. We find that $73 \%, 67 \%$, and 35\% of ICD9s for AMI, PNE, and HF, respectively, satisfy this criterion for non-deferrability. When we further restrict our analysis to patients admitted on an emergency or urgent basis, these percentages increase to $77 \%, 76 \%$, and 47\%, respectively. These results are consistent with Card et al. (2009), which finds that AMI and a number of PNE diagnoses satisfy the criterion for non-deferrability, implying that patient severity for these conditions does not differ by admission day-of-week. On the other hand, Card et al. (2009) did not find that HF, which is a chronic condition, satisfies the non-deferrability criterion. HF patients may have more discretion as to when to visit the hospital, suggesting that there may be a correlation between unobserved severity and admission day-of-week for these patients. As a result, we conclude that AMI and PNE are nondeferrable conditions, which strengthens our confidence in the validity of the instrument for these patients. However, because HF may be deferrable, this raises potential issues with our instrument for this group of patients. Because the percentage of ICD9s which satisfy the non-deferrability condition is higher when restricting to those admitted on an emergency or urgent basis, our analysis for all three conditions will be restricted to these admission categories.

A number of studies (e.g. Kc and Terwiesch (2012)) examine the exclusion criteria by comparing their IV to observable measures of severity. We do this as well and these t-tests corroborate our findings regarding non-deferrability. Specifically, we fail to reject the null hypothesis that the mean Elixhauser scores are not different on the IV days versus the other days for both AMI and PNE patients $(p=.21$ and $p=.75$, respectively). By this measure, there is no evidence that patients admitted on the IV days are different than those admitted on other days. The same is not true for HF patients. Hence, our main focus is the acute conditions, AMI and PNE. In Section 5, we consider the chronic condition, HF.

Relationship Between Admission Day-of-Week and LOS: Next, we examine whether admission dayof-week is correlated with our endogenous variable, LOS. Table 1 shows that the average LOS for AMI and PNE patients differs based on admission day-of-week. We estimated separate regressions of the logarithm of LOS on patient observables (age, gender, race, Elixhauser, DRG, had surgical procedure(s) or not), time dummies (month and year of hospital admission) and hospital fixed effects. Figure 1 shows the average residual from this regression plotted against the admission day-of-week for each patient cohort. For AMI patients, we can see that patients admitted on Monday or Tuesday have negative residuals, suggesting that they are 'prematurely' discharged. We see a similar effect for PNE patients, where patients admitted on Sunday, Monday or Tuesday have negative residuals.

\footnotetext{
${ }^{6}$ To account for potential differences across states, we run each t-test by state.
} 


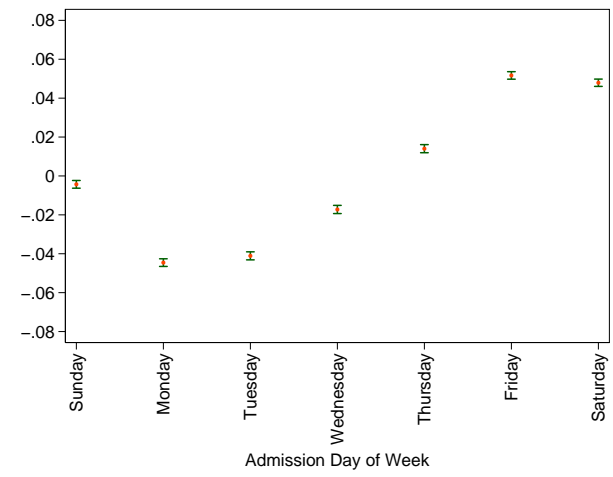

(a) AMI patients

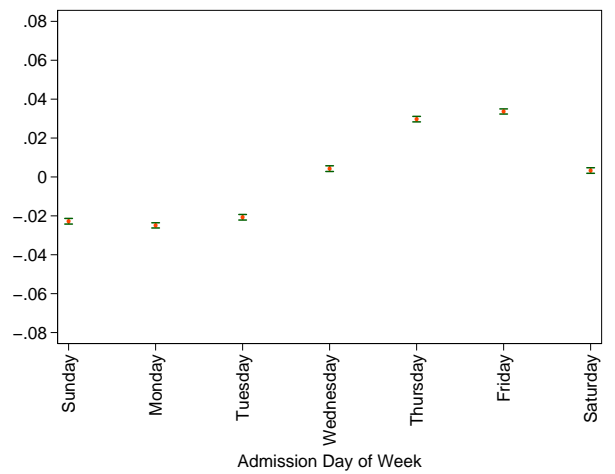

(b) PNE patients

Figure 1 Day-of-week effect: Average of residuals and their 95\% lower and upper bounds from regressions of $\log (L O S)$ on observables plotted against admission day-of-week

The average LOS for AMI and PNE patients in our sample is 5.4 and 5.5 days, respectively. As such, patients admitted on Sunday, Monday or Tuesday are likely to be ready for discharge on the weekend. There is substantial evidence (e.g. Varnava et al. (2002), Wong et al. (2009)) that hospitals prefer to discharge patients just prior to the weekend rather than keeping the patients over the weekend when many services are not available; this is also consistent with patients' preferences to be discharged, if possible, prior to the weekend ${ }^{7}$. Indeed, there seems to be evidence of this preference in our data where discharge rates peak on Friday and fall sharply on Saturday and Sunday (see Figure 2). This suggests that we may be able to leverage the variation in LOS due to this "discharge before the weekend effect" as an identification strategy and isolate a valid instrumental variable. Note that what we are considering a weekend effect is different than that seen in Rinne et al. (2014), which examines the impact of a weekend discharge on hospital readmissions and finds no effect. In contrast, we consider the effect of being discharged 'early' due to the hospitals' practice to discharge before the weekend. In results not reported here, we found that this admission dayof-week effect is observed even when stratifying by different levels of patient severity, as measured by the Elixhauser score.

Further details on the residuals of our $\log (L O S)$ regressions for AMI and PNE are shown in Figures 3 and 4, respectively, where the histograms of the residuals are plotted by admission day-of-week. An interesting observation from Figure 4 is that the histogram for Tuesday admissions is bi-modal, indicating that PNE patients admitted on Tuesday may be likely to be prematurely discharged prior to the weekend, or conversely, likely to be kept longer in the hospital, i.e. over the weekend to wait for further treatment and monitoring on Monday. The histograms in Figure 4 therefore suggest that Tuesday admission may not

\footnotetext{
${ }^{7}$ In discussions with administrators at a major medical center, we were informed that on the weekend social workers are generally not available and it is difficult to arrange for home health aides.
} 


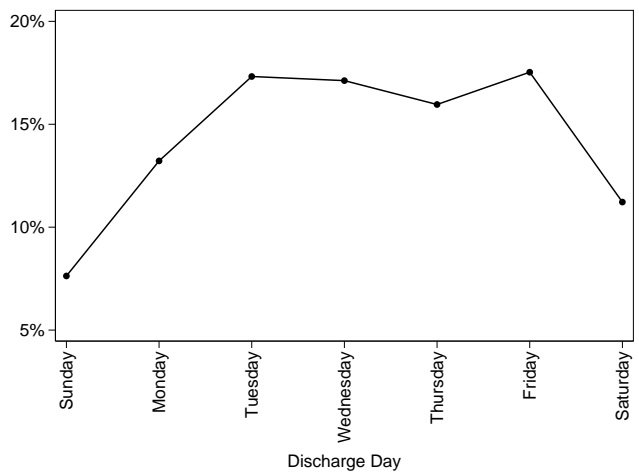

(a) AMI patients

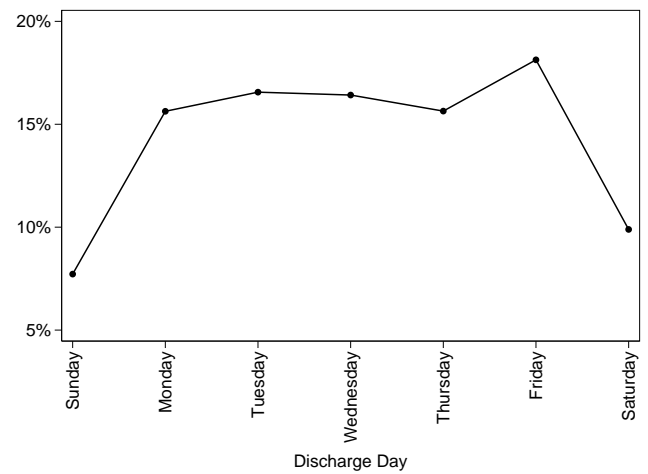

(b) PNE patients

Figure 2 Day-of-week effect: Percentage of discharges by day-of-week

be a useful instrument for PNE patients. In our main analysis for PNE patients, we drop those admitted on Tuesdays, so that the bi-modality of Tuesday admissions will not affect our estimates. As will be discussed next, we also drop patients admitted on the weekends, and use Monday admissions as the IV. We will test the sensitivity of the results to including patients admitted on Tuesday. We do not observe the bimodal behavior for Tuesday admissions for AMI patients, so we use Monday/Tuesday admissions as an instrument in the AMI model.

Resource Availability on IV days: The motivation for using day of admission as an instrument is that patients have shorter LOS when admitted earlier in the week because of the desire to discharge patients before the weekend. However, there might be concerns that the type of care provided to patients on weekends is different than other days of the week. This may be of particular concern for PNE patients, as Sunday admission could be considered as part of the instrumental variable. If patients admitted on Sunday receive different care than those admitted on other days of the week and if this is a primary driver of any differences in mortality we measure for Sunday/Monday admits, we may incorrectly attribute its effect to that of shorter LOS. For instance, with less staffing and resources available on weekends, one may question if patients admitted on Sundays have worse outcomes because of lack of access to care. Ryan et al. (2005) found that while cardiac patients admitted on the weekend have longer delays to catheterization, there does not seem to be any difference in outcomes. On the other hand, Dobkin (2003) found that weekend admission is associated with higher risk of mortality. As such, in our main analysis, we exclude weekend admits to address any concerns regarding differences in care provided on the weekend. This means that for PNE patients, Monday admits serve as the instrumental variable.

It is common for many surgeries, especially the more complex ones, to be scheduled on Mondays. Thus, while we focus on emergency and urgent patients, the availability of surgical staff may be reduced for 

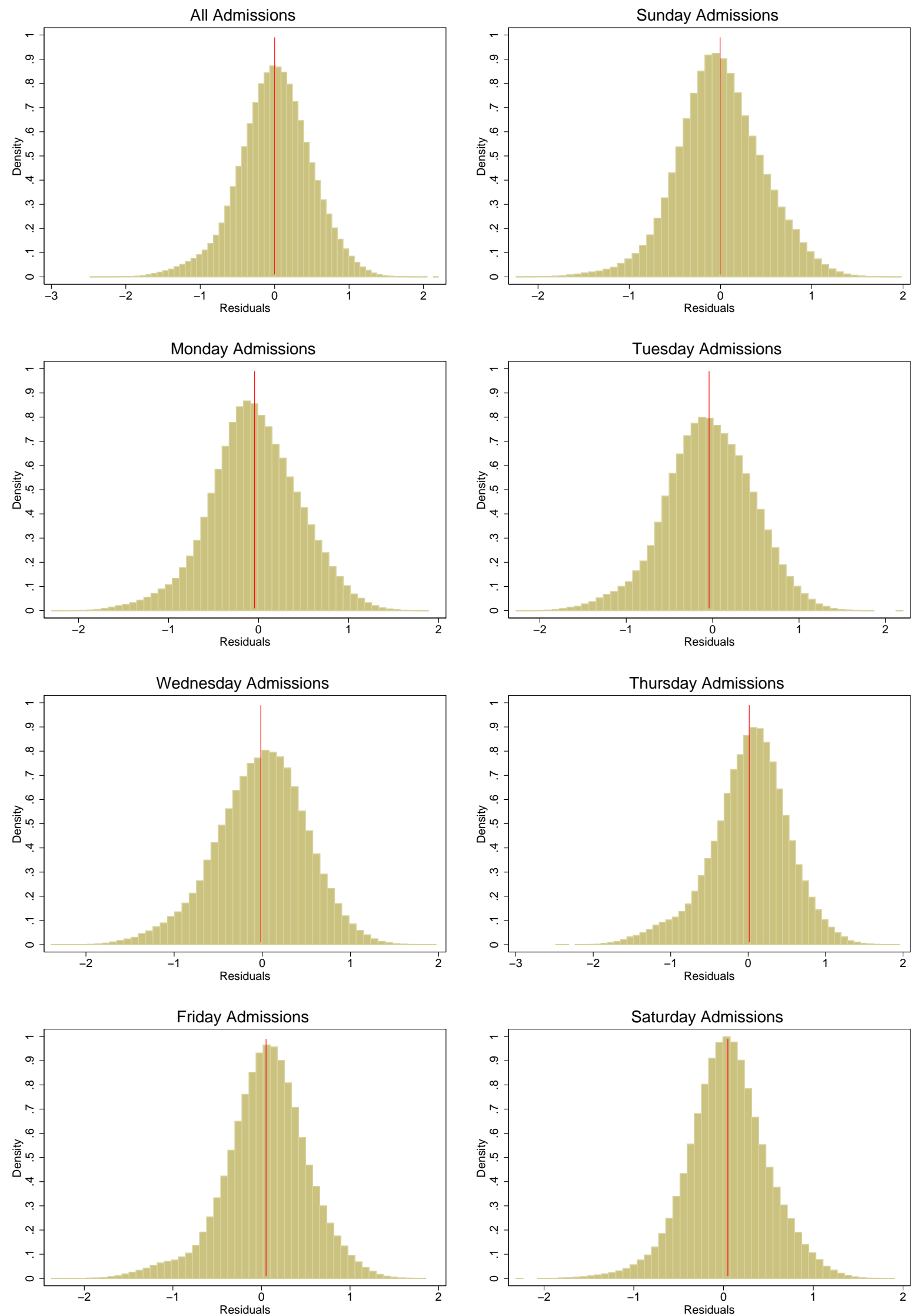

Figure 3 Residual histograms of $\log (L O S)$ regression for AMI patients by admission day-of-week 

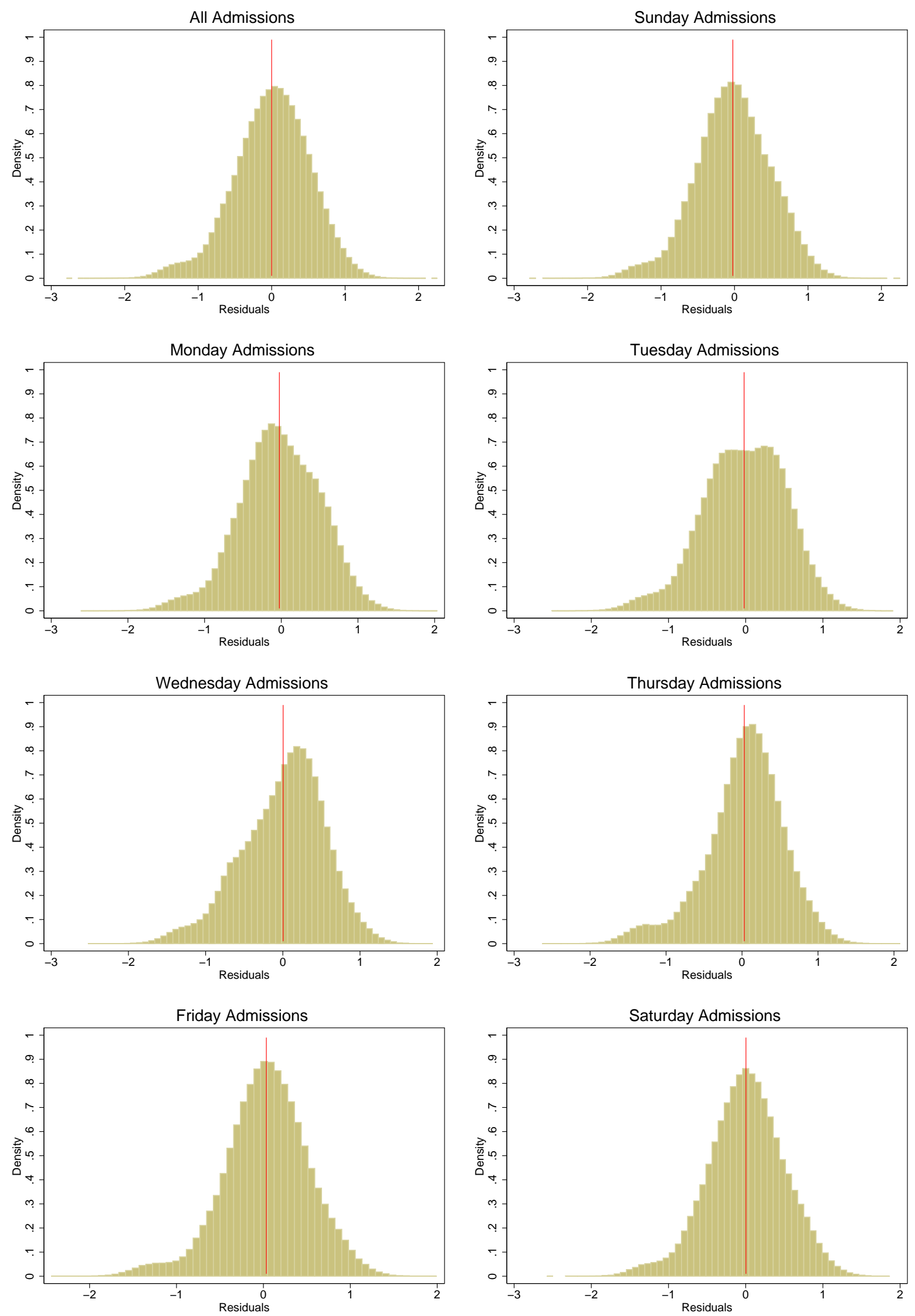

Figure 4 Residual histograms of $\log (L O S)$ regression for PNE patients by admission day-of-week 
patients admitted to a surgical service on Monday. As 71\% of AMI patients have some sort of surgical procedure, this may have an impact on their care. However, surgical schedules are unlikely to have a significant impact on care for patients admitted to a medical service. In Section 4.2, we will provide a robustness check which excludes patients who have a surgical procedure during their hospital stay.

\subsection{Estimation}

We now introduce our instrumental variable estimation approach. In the first stage, we fit a linear model for $\log (L O S)$ :

$$
\log \left(L O S_{i}\right)=\hat{\beta} X_{i}+\hat{\xi} M_{i}+\hat{\psi} Y R_{i}+\hat{\eta} H_{i}+\hat{\phi} Z_{i}+\nu_{i}
$$

In the second stage, Probit models for each of the binary patient outcomes, $D^{(t)}$ for $t \in\{3,7,30,60\}$, are estimated:

$$
\begin{aligned}
y_{i}^{*} & =\beta X_{i}+\theta \log \left(L O S_{i}\right)+\xi M_{i}+\psi Y R_{i}+\eta H_{i}+\epsilon_{i} \\
y_{i} & =1_{\left\{y_{i}^{*}>0\right\}}
\end{aligned}
$$

Thus, the first stage uses $Z_{i}$ as an instrument for $\log (L O S)$ in the second stage. For AMI patients, we let $Z_{i}$ be an indicator that equals to 1 if the patient is admitted on Monday or Tuesday, and 0 otherwise. Similarly, for PNE patients, $Z_{i}$ is an indicator that equals to 1 if the patient is admitted on Monday. These equations are estimated jointly via Maximum Likelihood Estimation (Wooldridge 2010). In some instances, the MLE estimation would not converge, which is a computational drawback of MLE noted in Wooldridge (2010), so we use the less efficient Newey's two-step estimator to estimate these equations. We estimate robust standard errors, clustered by admission day-of-week and year.

In examining the admission day-of-week effect in Equation (3), we expect the coefficient for our Monday/Tuesday and Monday admission day instrument $\left(Z_{i}\right), \hat{\phi}$, to be negative for the AMI and PNE cohorts, respectively. Finally, we hypothesize that these 'premature' discharges due to hospitals' desire to discharge patients before the weekend will increase the risk of mortality, so that $\theta$ is negative.

\section{Results for AMI and Pneumonia Patients}

We start by examining the estimation results for the acute conditions: AMI and PNE. In Section 5, we will consider the remaining condition, HF, which round out the three initial Hospital Compare conditions. 
Table 2 Mortality Model Results for AMI Patients

\begin{tabular}{|c|c|c|c|c|c|c|c|c|}
\hline & \multicolumn{4}{|c|}{ Probit } & \multicolumn{4}{|c|}{ IV (MonTue adm) Probit } \\
\hline & 3 days & 7 days & 30 days & 60 days & 3 days & 7 days & 30 days & 60 days \\
\hline \multicolumn{9}{|l|}{ Second Stage (Mortality) } \\
\hline \multirow[t]{2}{*}{$\log (\mathrm{LOS})$} & $-0.04 * * *$ & $0.02^{+}$ & $0.16 * * *$ & $0.21 * * *$ & $-0.23^{*}$ & $-0.19 *$ & $-0.18 * *$ & $-0.10^{+}$ \\
\hline & $(0.01)$ & $(0.01)$ & $(0.01)$ & $(0.01)$ & $(0.11)$ & $(0.09)$ & $(0.06)$ & $(0.06)$ \\
\hline Age, Gender, Race & Yes & Yes & Yes & Yes & Yes & Yes & Yes & Yes \\
\hline Elixhauser Categorical Vars & Yes & Yes & Yes & Yes & Yes & Yes & Yes & Yes \\
\hline Had surgical procedure(s) & Yes & Yes & Yes & Yes & Yes & Yes & Yes & Yes \\
\hline Month, Year Dummies & Yes & Yes & Yes & Yes & Yes & Yes & Yes & Yes \\
\hline Hospital FE & Yes & Yes & Yes & Yes & Yes & Yes & Yes & Yes \\
\hline \multicolumn{9}{|l|}{ First Stage $(\log (L O S))$} \\
\hline IV & & & & & $-0.06 * * *$ & $-0.06 * * *$ & $-0.06 * * *$ & $-0.06 * * *$ \\
\hline & & & & & $(0.00)$ & $(0.00)$ & $(0.00)$ & $(0.00)$ \\
\hline Age, Gender, Race & & & & & Yes & Yes & Yes & Yes \\
\hline Elixhauser Categorical Vars & & & & & Yes & Yes & Yes & Yes \\
\hline Had surgical procedure(s) & & & & & Yes & Yes & Yes & Yes \\
\hline Month, Year Dummies & & & & & Yes & Yes & Yes & Yes \\
\hline Hospital FE & & & & & Yes & Yes & Yes & Yes \\
\hline Num. of obs. & 1127156 & 1152272 & 1164446 & 1166601 & 1127156 & 1152272 & 1164446 & 1166601 \\
\hline Wald $\chi^{2}$ test & & & & & 3.03 & 5.33 & 28.73 & 28.59 \\
\hline Wald p-value & & & & & 0.08 & 0.02 & 0.00 & 0.00 \\
\hline
\end{tabular}

Table 3 Mortality Model Results for PNE Patients

\begin{tabular}{|c|c|c|c|c|c|c|c|c|}
\hline & \multicolumn{4}{|c|}{ Probit } & \multicolumn{4}{|c|}{ IV (Mon adm) Probit } \\
\hline & 3 days & 7 days & 30 days & 60 days & 3 days & 7 days & 30 days & 60 days \\
\hline \multicolumn{9}{|l|}{ Second Stage (Mortality) } \\
\hline $\log (\mathrm{LOS})$ & $\begin{array}{c}0.08 * * * \\
(0.01)\end{array}$ & $\begin{array}{c}0.14 * * * \\
(0.01)\end{array}$ & $\begin{array}{c}0.24 * * * \\
(0.01)\end{array}$ & $\begin{array}{c}0.25 * * * \\
(0.01)\end{array}$ & $\begin{array}{l}-0.24 * \\
(0.11)\end{array}$ & $\begin{array}{c}-0.29 * * * \\
(0.08)\end{array}$ & $\begin{array}{c}-0.23 * * * \\
(0.05)\end{array}$ & $\begin{array}{c}-0.09^{+} \\
(0.05)\end{array}$ \\
\hline Age, Gender, Race & Yes & Yes & Yes & Yes & Yes & Yes & Yes & Yes \\
\hline Elixhauser Categorical Vars & Yes & Yes & Yes & Yes & Yes & Yes & Yes & Yes \\
\hline Had surgical procedure(s) & Yes & Yes & Yes & Yes & Yes & Yes & Yes & Yes \\
\hline Month, Year Dummies & Yes & Yes & Yes & Yes & Yes & Yes & Yes & Yes \\
\hline Hospital FE & Yes & Yes & Yes & Yes & Yes & Yes & Yes & Yes \\
\hline \multicolumn{9}{|l|}{ First Stage $(\log (L O S))$} \\
\hline IV & & & & & $\begin{array}{c}-0.05 * * * \\
(0.00)\end{array}$ & $\begin{array}{c}-0.05 * * * \\
(0.00)\end{array}$ & $\begin{array}{c}-0.05 * * * \\
(0.00)\end{array}$ & $\begin{array}{c}-0.05 * * * \\
(0.00)\end{array}$ \\
\hline Age, Gender, Race & & & & & Yes & Yes & Yes & Yes \\
\hline Elixhauser Categorical Vars & & & & & Yes & Yes & Yes & Yes \\
\hline Had surgical procedure(s) & & & & & Yes & Yes & Yes & Yes \\
\hline Month, Year Dummies & & & & & Yes & Yes & Yes & Yes \\
\hline Hospital FE & & & & & Yes & Yes & Yes & Yes \\
\hline Num. of obs. & 2120417 & 2157226 & 2175717 & 2177974 & 2120417 & 2157226 & 2175717 & 2177974 \\
\hline Wald $\chi^{2}$ test & & & & & 7.96 & 25.74 & 93.99 & 55.06 \\
\hline Wald p-value & & & & & 0.00 & 0.00 & 0.00 & 0.00 \\
\hline
\end{tabular}

Note. Robust standard errors in parentheses. ${ }^{+}(p<0.1), *(p<0.05), * *(p<0.01), * * *(p<0.001)$.

\subsection{Mortality}

Since the main focus of our work is post-discharge mortality, we present the results for mortality here.

Readmissions are considered in the subsequent section because CMS posts both mortality and readmission 
rates on its Hospital Compare website.

Tables 2 and 3 show that when we do not instrument $\log (L O S)$, the coefficient of $\log (L O S)$ on the probability of mortality is positive and statistically significant at the $.1 \%$ level for all of the models, except for the AMI 3-day mortality model. For the AMI patients, when using Monday/Tuesday admission to instrument $\log (L O S)$, the coefficient on $\log (\mathrm{LOS})$ is negative and significant in all four mortality models. The $\mathrm{F}$ statistic for the significance of the instrument in the first-stage regression is 3933.01 for the AMI patients, which indicates our instrument is quite strong. The average marginal effect of a one-day increase in LOS is a reduction in the 30-day mortality risk from .0740 to .0694 , which is about a $6 \%$ decrease. The marginal effect varies from a $3 \%$ decrease for 60 -day mortality up to a $11 \%$ decrease for 3-day mortality. We will use these estimates of the marginal effects when considering different patient care strategies in Section 7 . The marginal effects are summarized in Tables 9 and 10.

For PNE patients, in Table 3, we again find that in all specifications with the Monday admits as an instrumental variable, we have a negative and statistically significant coefficient on $\log (L O S)$. The $\mathrm{F}$ statistic for the significance of the instrument in the first-stage regression is 3588.08 for the PNE patients, which again indicates that our instrument is quite strong. Note that, as seen in Table 4, these results are robust to including patients admitted on Tuesday, whose distribution for residual LOS exhibited bi-modal behavior (Figure 4). For 60-day mortality, we have weak $(p<.10)$ evidence that $\log (L O S)$ has an impact for AMI (or PNE). Recall that AMI and PNE are acute conditions and we are examining the impact of an additional day in the hospital. We expect that if there is an impact, it will be most substantial for mortality in shorter time windows. Our results partially support this. For AMI patients, the magnitude of the impact of an additional day in the hospital on post-discharge mortality is decreasing as the time window increases. For PNE patients, this monotonicity is violated by the 3-day mortality measure, but holds for the remaining time windows.

Table 4 Mortality Model Results for PNE Patients including Tuesday admits

\begin{tabular}{l|cccc}
\hline & 3 days $\dagger$ & 7 days $\dagger$ & 30 days $\dagger$ & 60 days \\
\hline $\log (\mathrm{LOS})$ & $-0.26^{+}$ & $-0.37 * *$ & $-0.28 * * *$ & -0.09 \\
& $(0.15)$ & $(0.12)$ & $(0.08)$ & $(0.06)$ \\
\hline $\mathrm{IV}$ & $-0.04 * * *$ & $-0.04 * * *$ & $-0.04 * * *$ & $-0.04 * * *$ \\
& $(0.00)$ & $(0.00)$ & $(0.00)$ & $(0.00)$ \\
\hline Num. of obs. & 2669589 & 2702151 & 2720306 & 2722223 \\
Wald $\chi^{2}$ test & 5.12 & 18.17 & 40.58 & 34.42 \\
Wald p-value & 0.02 & 0.00 & 0.00 & 0.00 \\
\hline Note. Robust standard errors in parentheses. ${ }^{+}(p<0.1), *(p<$ \\
$0.05), * *(p<0.01), * * *(p<0.001) . \dagger$ Estimated using two-step esti- \\
mator.
\end{tabular}

The results of the Wald $\chi^{2}$ test suggest that our instrument is able to control for a substantial portion of the endogeneity bias in our sample. This, along with the non-deferrability results (Card et al. 2009) and the 
results of our t-tests comparing the Elixhauser scores of AMI (PNE) patients admitted on Monday/Tuesday (Monday) versus other days, supports the reliability of the IV estimates.

\subsection{Robustness Checks}

Tables 5 and 6 present a number of robustness checks for the mortality model. For the sake of space, we only present results for 30-day mortality, which is the measure reported in Hospital Compare, in the main body of the paper. The results for the remaining three mortality measures can be found in the Appendix.

The first issue that we address is the potential that the ICD9 and DRG codes (used to indicate the patients' conditions and severity) are inaccurate due to potential upcoding hospitals may utilize to increase Medicare payments, which are provided on a Fee-for-service basis. To address this, we restrict our analysis to nonprofit hospitals because they have little incentive to upcode (see the discussion in Powell et al. (2012)); the results are reported in Column 1 of Table 5 for AMI patients and Table 6 for PNE patients. In Column 2, we restrict the analysis to hospitals that are in the top quartile for number of patients as one might anticipate that the effects of LOS on mortality could be different for these hospitals that are more likely to treat complex cases. In Column 3, we exclude patients with a surgical procedure during their hospital stay as an increase in complex scheduled surgeries on Mondays may reduce the availability of staff to treat the emergency and urgent patients who are admitted to a surgical service. In Column 4, we include patients who are admitted on the weekends. Note that for PNE patients, this means our instrument is Sunday/Monday admits (instead of just Monday admits). Finally, in Column 5, we randomly select one hospital encounter per patient. This is because an individual patient may have multiple admissions, which are each counted as a separate observation, in our sample; about $30 \%$ of our observations are from patients with multiple visits during our study period. Thus, we include a robustness check that only includes a single hospitalization per patient. In all five columns of Tables 5 and 6, the coefficient on $\log (L O S)$ is negative and the results of the Wald $\chi^{2}$ test suggest that our instrument is able to control for some of the endogeneity. For AMI patients, the coefficient on $\log (L O S)$ is no longer statistically significant when restricting to non-profit hospitals or when including weekend admits. For PNE patients, all robustness checks still find a statistically significant effect of $\log (L O S)$ on mortality.

\section{Results for Heart Failure Patients}

We now extend our analysis to the HF patients. Summary statistics for these patients are shown in Table 1.

In Section 3.1, we used the residual plots in Figure 1 to determine an IV based on admission day-of-week for $\log (L O S)$ for the AMI and PNE patients. Similarly, Figure 5(a) plots residuals from a regression of $\log (L O S)$ on patient observables and seasonality and hospital fixed effects against admission day-of-week for the HF patients. Similar to PNE, we found that patients admitted on Sunday, Monday and Tuesday had 
Table 5 Robustness Check of Mortality in $\mathbf{3 0}$ days Model Results for AMI Patients

\begin{tabular}{l|ccccc}
\hline & Non-profit & Big & $\begin{array}{c}\text { w/o Surgical } \\
\text { Procedures }\end{array}$ & $\begin{array}{c}\text { w/ Weekend } \\
\text { Admits }\end{array}$ & $\begin{array}{c}\text { Random } \\
\text { Episode }\end{array}$ \\
\hline $\log (\mathrm{LOS})$ & -0.10 & $-0.19 * *$ & $-0.21 *$ & -0.09 & $-0.20 * *$ \\
& $(0.08)$ & $(0.07)$ & $(0.08)$ & $(0.06)$ & $(0.07)$ \\
\hline IV & $-0.06 * * *$ & $-0.06 * * *$ & $-0.07 * * *$ & $-0.06 * * *$ & $-0.06 * * *$ \\
& $(0.01)$ & $(0.01)$ & $(0.00)$ & $(0.00)$ & $(0.00)$ \\
\hline Num. of obs. & 824970 & 923721 & 332934 & 1597921 & 1063274 \\
Wald $\chi^{2}$ test & 12.98 & 30.04 & 10.11 & 18.47 & 27.15 \\
Wald p-value & 0.00 & 0.00 & 0.00 & 0.00 & 0.00 \\
\hline Note. Robust standard errors in parentheses. ${ }^{+}(p<0.1), *(p<0.05), * *(p<0.01), * * *(p<$ \\
$0.001)$.
\end{tabular}

Table 6 Robustness Check of Mortality in $\mathbf{3 0}$ days Model Results for PNE Patients

\begin{tabular}{l|ccccc}
\hline & Non-profit $\dagger$ & Big & $\begin{array}{c}\text { w/o Surgical } \\
\text { Procedures }\end{array}$ & $\begin{array}{c}\text { w/ Weekend } \\
\text { Admits }\end{array}$ & $\begin{array}{c}\text { Random } \dagger \\
\text { Episode }\end{array}$ \\
\hline $\log (\mathrm{LOS})$ & $-0.22^{* *}$ & $-0.23^{* * *}$ & $-0.28^{* * *}$ & $-0.33^{* * *}$ & $-0.29 * * *$ \\
& $(0.08)$ & $(0.05)$ & $(0.06)$ & $(0.05)$ & $(0.07)$ \\
\hline IV & $-0.05^{* * *}$ & $-0.05^{* * *}$ & $-0.05 * * *$ & $-0.04 * * *$ & $-0.05 * * *$ \\
& $(0.00)$ & $(0.00)$ & $(0.00)$ & $(0.00)$ & $(0.00)$ \\
\hline Num. of obs. & 1421575 & 1371076 & 1581974 & 3139668 & 1829183 \\
Wald $\chi^{2}$ test & 36.71 & 74.58 & 70.40 & 108.55 & 57.50 \\
Wald p-value & 0.00 & 0.00 & 0.00 & 0.00 & 0.00 \\
\hline
\end{tabular}

shorter LOS. We also generated residual histograms similar to Figures 3 and 4, which can be seen in Figure A.1 in the Appendix. There is evidence of bi-modality for Tuesday admissions for the HF patients, so we exclude Tuesday admits in our main model. Similar to the findings for the AMI and PNE patients, Figure 5(b) shows that discharge rates for HF patients peak on Friday and fall sharply on Saturday and Sunday. Based on our observations, we use Monday admissions as an instrument in the HF model since we exclude weekend and Tuesday admits.

We again use t-tests to compare the mean Elixhauser scores of HF patients admitted on Monday versus the other days of the week. We reject the null hypothesis $(p<.001)$ that patients admitted on Monday have the same mean Elixhauser score as those admitted on non-IV days. In fact, we fail to reject the null hypothesis $(p=1.00)$ that those admitted on Monday have higher Elixhauser score than those admitted on other days. This makes it difficult to assess whether any increase in mortality risk is because patients admitted on Monday are sicker or because they had a shorter LOS due to the 'discharge before the weekend' effect. In other words, the results regarding the Elixhauser score could invalidate our proposed IV. This is not too surprising as HF is a chronic condition; due to its chronic nature, HF patients may have more discretion deciding when to go to the hospital. Also recall that AMI and PNE were identified as 'nondeferrable diagnoses' using the approach in Card et al. (2009), while HF was not. Our instrument is, hence, 


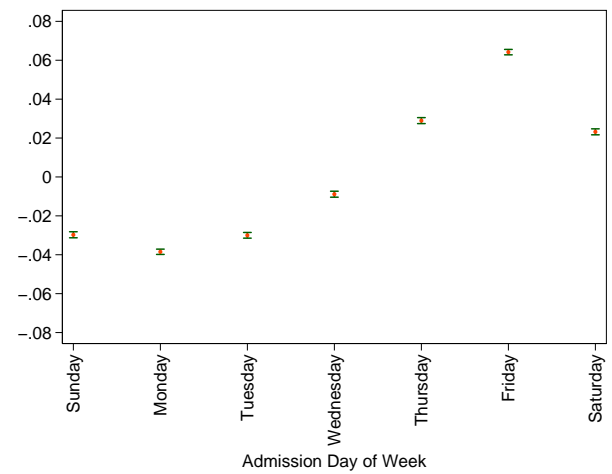

(a)Average of residuals and their $95 \%$ lower and upper bounds from regression of $\log (L O S)$ on observables plotted against admission day-of-week

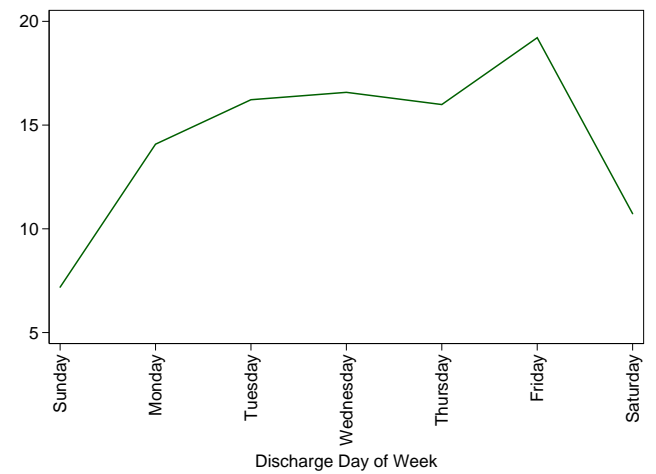

(b)Percentage of discharges by day-of-week

Figure 5 Day-of-week effects on LOS for HF patients

unlikely to be valid for this group of patients. Nonetheless, for completeness we examine the results for HF.

Table 7 shows our main results for all four mortality measures for HF patients. As with AMI and PNE, we see that when we do not instrument $\log (L O S)$, the coefficient of $\log (L O S)$ is positive and statistically significant. When we use Monday admission day as an instrument, all coefficients are negative and statistically significant. The F statistic for the significance of the instrument in the first-stage regression is 6541.77. Moreover, the results of the Wald $\chi^{2}$ test suggest that the instrument is able to control for some of the endogeneity. As discussed above, we have some concerns regarding the validity of this instrument. While the results suggest that an additional day in the hospital can reduce mortality risk for HF patients, their reliability is uncertain due to potential issues with the instrumental variable.

\section{Readmission results}

While the focus of our analysis has been on the impact of an additional hospital day on mortality risk, we also examine 30-day readmissions. We do this for two primary reasons: 1) readmissions is the second outcome measure reported on Hospital Compare and 2) there has been substantial focus on 30-day readmission due to the Hospital Readmission Reduction Program which is part of the Affordable Care Act.

The analysis is similar to that described in Section 3.2, where $y_{i}$ is now an indicator for 30-day readmissions. We use the same instrumental variables identified previously: Monday admits for PNE and HF and Monday/Tuesday admits for AMI. Previous studies of readmissions have used different IVs. Focusing on HF patients in California, Makowsky and Klein (2015) use hospital occupancy rates and emergency vehicle diversions, both of which are only publicly available for the state of California. Carey (2015) uses number of procedures for her study of AMI patients in New York State but this is an invalid instrument for our 
Table 7 Mortality Model Results for HF Patients

\begin{tabular}{|c|c|c|c|c|c|c|c|c|}
\hline & \multicolumn{4}{|c|}{ Probit } & \multicolumn{4}{|c|}{ IV (Mon adm) Probit } \\
\hline & 3 days & 7 days & 30 days & 60 days & 3 days & 7 days & 30 days & 60 days \\
\hline \multicolumn{9}{|l|}{ Second Stage (Mortality) } \\
\hline $\log (\mathrm{LOS})$ & $\begin{array}{c}0.20 * * * \\
(0.01)\end{array}$ & $\begin{array}{c}0.23 * * * \\
(0.01)\end{array}$ & $\begin{array}{c}0.29 * * * \\
(0.01)\end{array}$ & $\begin{array}{c}0.30 * * * \\
(0.00)\end{array}$ & $\begin{array}{l}-0.16^{*} \\
(0.06)\end{array}$ & $\begin{array}{l}-0.12 * \\
(0.06)\end{array}$ & $\begin{array}{l}-0.10 * \\
(0.04)\end{array}$ & $\begin{array}{l}-0.07 * \\
(0.03)\end{array}$ \\
\hline Age, Gender, Race & Yes & Yes & Yes & Yes & Yes & Yes & Yes & Yes \\
\hline Elixhauser Categorical Vars & Yes & Yes & Yes & Yes & Yes & Yes & Yes & Yes \\
\hline Had surgical procedure(s) & Yes & Yes & Yes & Yes & Yes & Yes & Yes & Yes \\
\hline Month, Year Dummies & Yes & Yes & Yes & Yes & Yes & Yes & Yes & Yes \\
\hline Hospital FE & Yes & Yes & Yes & Yes & Yes & Yes & Yes & Yes \\
\hline \multicolumn{9}{|l|}{ First Stage $(\log (L O S))$} \\
\hline IV & & & & & $\begin{array}{c}-0.07 * * * \\
(0.00)\end{array}$ & $\begin{array}{c}-0.07 * * * \\
(0.01)\end{array}$ & $\begin{array}{c}-0.07 * * * \\
(0.01)\end{array}$ & $\begin{array}{c}-0.07 * * * \\
(0.01)\end{array}$ \\
\hline Age, Gender, Race & & & & & Yes & Yes & Yes & Yes \\
\hline Elixhauser Categorical Vars & & & & & Yes & Yes & Yes & Yes \\
\hline Had surgical procedure(s) & & & & & Yes & Yes & Yes & Yes \\
\hline Month, Year Dummies & & & & & Yes & Yes & Yes & Yes \\
\hline Hospital FE & & & & & Yes & Yes & Yes & Yes \\
\hline Num. of obs. & 2376119 & 2433290 & 2457298 & 2459456 & 2376119 & 2433290 & 2457298 & 2459456 \\
\hline Wald $\chi^{2}$ test & & & & & 28.85 & 39.50 & 82.19 & 126.05 \\
\hline Wald p-value & & & & & 0.00 & 0.00 & 0.00 & 0.00 \\
\hline
\end{tabular}

sample because the correlation between the number of procedures and the residuals of the mortality model is statistically different from zero.

Table 8 summarizes our results. We find no statistically significant results for AMI and HF. Interestingly, we find a statistically significant positive coefficient on $\log (L O S)$ for PNE patients. However, upon closer examination, we see that the Wald $\chi^{2}$ test for PNE patients suggests that for readmissions, our instrument is not effective at addressing the endogeneity concern. As such, the positive coefficient is likely due to our inability to adequately control for unobserved biases.

Table 8 Readmission in 30 days Model Results

\begin{tabular}{l|ccc}
\hline & AMI & PNE & HF \\
\hline $\log (\mathrm{LOS})$ & 0.03 & $0.12^{*}$ & 0.00 \\
& $(0.04)$ & $(0.06)$ & $(0.03)$ \\
\hline IV & $-0.06 * * *$ & $-0.05 * * *$ & $-0.07 * * *$ \\
& $(0.00)$ & $(0.00)$ & $(0.01)$ \\
\hline Num. of obs. & 1074784 & 2005748 & 2274860 \\
Wald $\chi^{2}$ test & 8.92 & 0.00 & 10.62 \\
Wald p-value & 0.00 & 0.98 & 0.00 \\
\hline Note. Robust standard errors in parentheses. ${ }^{+}(p<0.1), *(p<$ \\
$0.05), * *(p<0.01), * * *(p<0.001)$.
\end{tabular}

It is not too surprising that we find little evidence that an extra day in the hospital impacts 30-day readmissions. This is consistent with the findings of Jaeker and Tucker (2015). Additionally, many critics of the 
Hospital Readmission Reduction Program point out that "the primary drivers of variability in 30-day readmission rates are the composition of a hospital's patient population and the resources of the community in which it is located-factors that are difficult for hospitals to change" (Joynt and Jha 2012), which is further corroborated in Carey and Lin (2014).

\section{Policy Implications}

In this section, we utilize our results from Section 4 to estimate the impact of various policy changes that hospitals or CMS can implement to reduce post-discharge deaths. In our analysis, we take the perspective of the social planner who aims to reduce adverse outcomes and overall costs. Having observed that keeping a patient in the hospital for one more day is an effective intervention to reduce 3-, 7-, 30-, and 60-day mortality, we compare the following two policies: (1) Keep the status quo or (2) Increase LOS by one day. This allows us to compare the effect of inpatient care on post-discharge mortality. Due to our concerns with the results for HF, we focus only on AMI and PNE patients.

To compare the cost-effectiveness of these two policies, we first discuss the cost estimates we will use. Taheri et al. (2000) estimate the cost of an additional day in the hospital to be $\$ 420$ in 1998 , which is $\$ 610$ in 2014 when adjusted for inflation. Importantly, Taheri et al. (2000) show that the direct cost of the last day represents only $2.4 \%$ of the total hospitalization cost. The Henry J. Kaiser Family Foundation (2014) provides an alternative measure and reports that the average hospital expenses for a day of inpatient care in the U.S. was $\$ 1,960$ in 2011, or $\$ 2,094$ in 2014 dollars. However, this measure includes an adjustment for outpatient care and is therefore likely to be an overestimate of the actual costs of inpatient care. Based on these two references, we assume that the cost of keeping a patient in the hospital one more day is $\$ 610$ or $\$ 2,094$, depending on whether one uses the marginal or average cost estimate. We note that the cost of a hospital day may also change over time according to other factors besides inflation; an example is the introduction of more costly procedures and tests. This change could be more or less relative to overall inflation, so we will also consider the robustness of our results to various rates of increase between 1998 and 2014 for the Taheri et al. (2000) cost estimates and between 2011 and 2014 for the The Henry J. Kaiser Family Foundation (2014) cost estimates.

Next, we use the estimates provided in Murphy and Topel (2006) for the benefits of reduced mortality. They calculated the value of a life-year for an average 80 year-old (the approximate mean age of the patients in our sample) to be $\$ 150,000$ per person in 1999 , which translates to $\$ 214,492$ per year or $\$ 17,874$ per month in 2014 dollars. Recognizing this may be an overestimate for individuals with serious medical conditions, we also consider how robust our insights are to alternative value of life estimates. 


\subsection{AMI Patients}

Starting with AMI patients, Table 9 summarizes the estimated mortality rates under the aforementioned two policies, constructed using the results for all mortality measures from Table 2 . We see that the reduction in mortality risk due to an additional day in the hospital ranges from $3 \%$ to $10.6 \%$, with the largest impact on 3-day mortality.

For ease of exposition when considering our policy change, we focus on 30-day mortality which is the reported Hospital Compare outcome measure. Over the 12 years in our data, there were 1,600,420 AMI patients (see Table 1). Therefore, we estimate that on average there are 133,368 AMI patients treated each year. With a $7.40 \%$ baseline mortality rate, this would correspond to 9,869 deaths each year. An increase of one day in the LOS of all AMI patient results in a mortality rate of $6.94 \%$, or a total of 9,256 deaths. That is, the inpatient intervention saves an additional 613 lives annually for the cohort we study compared to the baseline.

Table 9 Estimated mortality percentages of AMI patients.

\begin{tabular}{l|cccc}
\hline & 3 days & 7 days & 30 days & 60 days \\
\hline No change (baseline) & $\mathbf{1 . 7 0}$ & $\mathbf{3 . 0 6}$ & $\mathbf{7 . 4 0}$ & $\mathbf{1 0 . 6 2}$ \\
Increase LOS by 1 day & 1.52 & 2.81 & 6.94 & 10.30 \\
\hline Absolute change & -0.18 & -0.25 & -0.46 & -0.32 \\
Percent change & $-10.6 \%$ & $-8.2 \%$ & $-6.2 \%$ & $-3.0 \%$ \\
\hline
\end{tabular}

We explore whether keeping the AMI patients in the hospital an extra day is cost-effective over the baseline of doing nothing. If hospitals kept all 133,368 AMI patients for one more day, the extra costs would range from $\$ 81,354,480$ (using Taheri's estimate of the marginal cost of an extra day) to $\$ 279,272,592$ (using Kaiser's estimate of the average cost of a hospital day). Since we are saving 613 lives as a result of this intervention, the total value of these saved lives is $\$ 17,874 \times 613=\$ 10,956,762$ for each month these patients live when we use Murphy and Topel (2006) to estimate the value of an additional month. This means that the patients would need to live $\$ 81,354,480 / \$ 10,956,762=7.4$ months (using the marginal cost estimate) or 25.5 months (using the average cost estimate) in order for the inpatient intervention to be cost-effective over the baseline of doing nothing. In our data, we find that AMI patients who survive for 30 days after hospital discharge live for another 5.8 years (69.5 months) on average ${ }^{8}$, suggesting that the inpatient intervention is cost-effective.

We next consider the robustness of the cost-effectiveness of our proposed intervention. In particular, since the average survival of AMI patients is 69.5 months, we consider the range of rates of increase in cost

\footnotetext{
${ }^{8}$ Note that our estimates for average survival are conservative as our data are truncated with the last recorded date of death being December 26, 2012; for any patient missing a date of death (i.e., they did not die before 12/26/2012) we assigned a death date of December 26, 2012.
} 
and the range in reductions in the value of living an additional month for which it is still cost-effective to keep patients an additional day. That is, if the percentage increase in cost is $x$, then the marginal cost of an additional day in 2014 would be $\$ 420 \times(1+x)^{16}$. The area under the curve in Figure 6(a) demonstrates the regime where it is cost-effective to keep patients an additional day in the hospital when costs are given by the marginal cost from Taheri et al. (2000). The circle indicates the case where the cost of a hospital day is adjusted by inflation only (to $\$ 610$ in 2014 dollars). We can see in this case the cost-effectiveness of keeping a patient an additional day is robust to reductions in the value of living an additional month of up to $89 \%$ of the estimates from Murphy and Topel (2006). It is also robust up to increases in annual cost of $17.7 \%$. Further reductions in the benefits of living an additional month and/or larger annual increases in cost would render the baseline as more cost-effective. Figure 6(b) shows the same for when the average cost estimate of an additional hospital day is used. We see that the cost-effectiveness of keeping patients in the hospital an additional day is very robust.

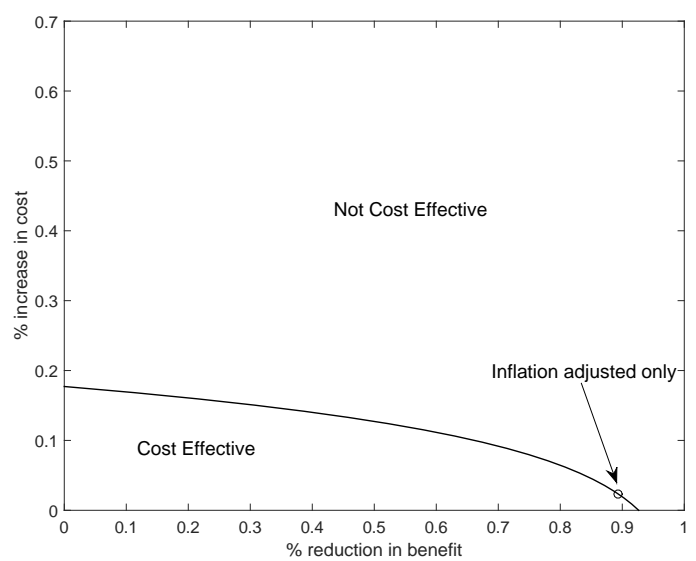

(a) Taheri et al. (2000) costs

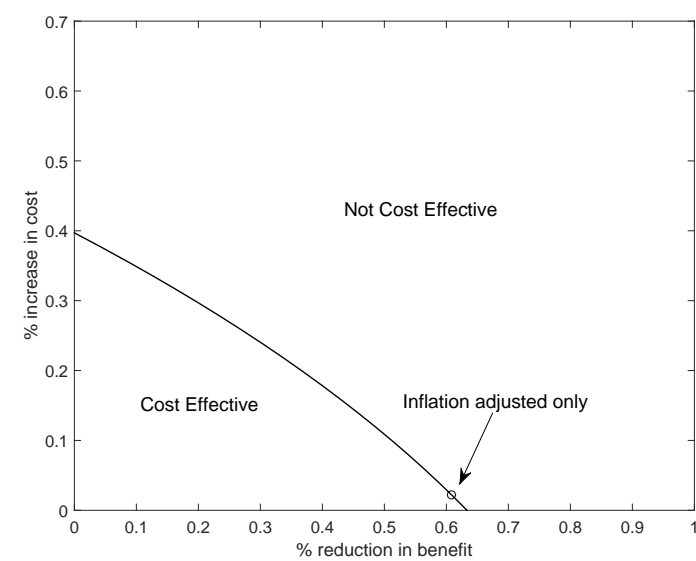

(b) The Henry J. Kaiser Family Foundation (2014) costs

Figure 6 AMI patients: Robustness of cost-effectiveness for keeping patients in the hospital an additional day. Baseline benefits are given by the value of living an additional month as estimated in Murphy and Topel (2006).

\subsection{Pneumonia Patients}

Table 10 uses the results from Table 3 and reports the estimated mortality rates for PNE patients under the baseline and the policy of increasing all patients' LOS by one day. We again see that keeping patients an additional day can be extremely effective for reducing mortality. For the 3,686,544 PNE patients in our twelve year cohort, increasing their LOS by one day saves 1,966 lives per year. Following the same methodology described above, we calculate the extra costs of keeping the PNE patients in the hospital for one more day to range from $\$ 187,399,320$ (using the marginal cost estimate) to $\$ 643,301,928$ (using 
the average cost estimate) per year. In addition, the benefits of saving 1,966 lives is $\$ 35,140,284$ for each month the patients live. This means that the patients would need to live 5.3 months (using the marginal cost estimate) or 18.3 months (using the average cost estimate) for the inpatient intervention to be cost-effective over doing nothing. In our data, we find that PNE patients who survive for 30 days after hospital discharge live for another 5.5 years (66.1 months) on average, making the inpatient intervention highly cost-effective.

Table 10 Estimated mortality percentages of PNE patients.

\begin{tabular}{l|cccc}
\hline & 3 days & 7 days & 30 days & 60 days \\
\hline No change (baseline) & $\mathbf{1 . 4 0}$ & $\mathbf{2 . 8 2}$ & $\mathbf{8 . 0 3}$ & $\mathbf{1 1 . 9 1}$ \\
Increase LOS by 1 day & 1.25 & 2.46 & 7.39 & 11.59 \\
\hline Absolute change & -0.15 & -0.36 & -0.64 & -0.32 \\
Percent change & $-10.7 \%$ & $-12.8 \%$ & $-8.00 \%$ & $-2.7 \%$ \\
\hline
\end{tabular}

As seen in Figure 7, the cost-effectiveness of the inpatient intervention is robust to reductions in the value of living an additional month of up to $91.9 \%$ (using the marginal cost estimate of a hospital day) or $70.4 \%$ (using the average cost estimate of a hospital day). It is also robust to high rates of increase in cost of nearly $20 \%$ (using the marginal cost) or 53\% (using the average cost). In sum, keeping PNE patients in the hospital one more day seems to be a cost-effective intervention for reducing 30-day mortality rates. These results are robust to the possibility that Murphy and Topel (2006) vastly overestimates the value of life for our average patient as well as the possibility that hospital costs are increasing at a rate much faster than inflation.

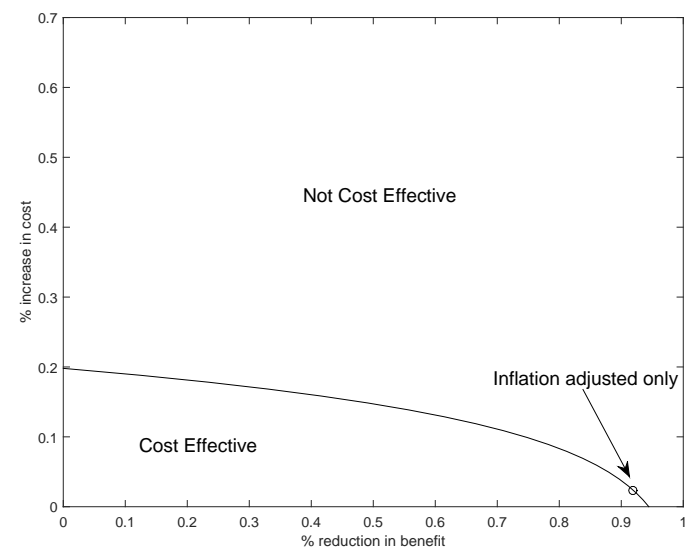

(a) Taheri et al. (2000) costs

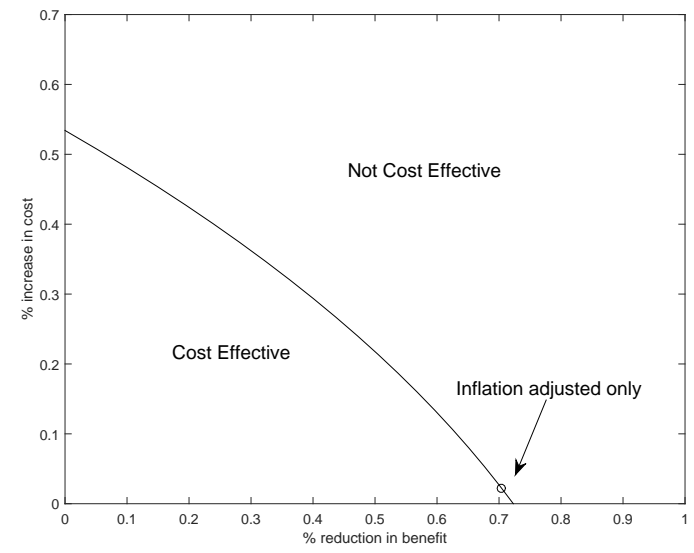

(b) The Henry J. Kaiser Family Foundation (2014) costs

Figure 7 PNE patients: Robustness of cost-effectiveness for keeping patients in the hospital an additional day. Baseline benefits are given by the value of living an additional month as estimated in Murphy and Topel (2006). 


\subsection{Managerial Implications}

Our policy analysis suggests that keeping AMI and PNE patients in the hospital one more day is costeffective from a social planner's perspective. Over the past few years, the U.S. government and CMS have taken steps through legislation, e.g. the Affordable Care Act, to provide incentives to healthcare providers to improve quality of care. Therefore, it is useful to consider the operational changes that hospitals would need to introduce if CMS were to require them to keep patients in the hospital a day longer. Since we have documented that premature discharges occur right before the start of the weekend, our analysis suggests that one way to reduce the number of patients who are discharged too early is for hospitals to discharge patients 7-days a week rather than preferentially discharging Monday through Friday. In order to move to a 7-day-a-week discharge cycle, hospital managers would need to provide sufficient staff on the weekends, notably social workers and others who facilitate the discharge process. This would enable patients to avoid premature discharges due to hospitals' desire to discharge before the weekend.

We note that premature discharges may arise for other reasons (e.g. congestion in inpatient beds (Kc and Terwiesch 2012, 2009), family/patient preferences, etc.) and our results suggest that hospitals should also take steps to avoid these types of discharges whenever possible. For instance, hospital administrators should consider the potential benefits of increasing bed capacity if congestion in inpatient beds appears to be a frequent initiator for early discharges. Separately, when faced with pressure to discharge a patient early, physicians and social workers should educate patients and family members and emphasize the potential benefits of remaining in the hospital an extra day.

\section{Conclusions}

In this paper, we examine the potential reductions in post-discharge mortality due to keeping patients in the hospital longer. Using an instrumental variables methodology and a dataset from CMS that consists of all Medicare FFS in-hospital patient visits between 2000 and 2011, we find that: (1) Keeping all pneumonia (PNE) patients in the hospital for one more day would save 1,966 lives per year for the cohort we study and the value of these saved lives exceeds the cost of the extra hospital day under reasonable assumptions, (2) Keeping all heart attack (AMI) patients in the hospital for one more day saves an additional 613 lives per year for the cohort we study and the value of these saved lives exceeds the cost of the extra hospital day under reasonable assumptions, and (3) While we see some evidence that an extra day may reduce mortality for heart failure patients, the reliability of these results is somewhat uncertain. The fact that we find compelling evidence that keeping AMI and PNE patients in the hospital for one more day significantly decreases their mortality rates shows that there are factors within a hospital's control, i.e. LOS, that impact post-discharge mortality; therefore, the 30-day mortality measures reported by Hospital Compare are indeed 
reasonable indicators of hospital quality and the government should explore how to more widely disseminate the information available on the Hospital Compare website.

Although we use a very comprehensive database, we excluded elective patients from our analysis because our instrument for LOS, day-of-week on which the patient was admitted, is most valid for patients admitted on an emergency or urgent basis. Hence, one limitation of our study is that the results may not apply to elective patients. A second limitation is that, while we provide convincing evidence that an extra day in the hospital significantly reduces mortality risk for heart attack and pneumonia patients, we do not know exactly why the extra day is beneficial. An extra day may provide more time for patients to be educated about their post-discharge behavior and/or it may enable the patient to reach a higher level of stability. Future research should explore the underlying causes of the relationship between hospital LOS and post-discharge mortality, which can help hospitals to improve their quality of care.

\section{References}

Baiocchi, M., J. Cheng, D. S. Small. 2014. Instrumental variable methods for causal inference. Statistics in Medicine 33(13) 2297-2340.

Bueno, H., J. S. Ross, Y. Wang, J. Chen, M. T. Vidán, S. T. Normand, J. P. Curtis, E. E. Drye, J. H. Lichtman, P. Keenan, M. Kosiborod, H. M. Krumholz. 2010. Trends in length of stay and short-term outcomes among medicare patients hospitalized for heart failure, 1993-2006. JAMA: The Journal of the American Medical Association 303(21) 2141-2147.

Card, D., , C. Dobkin, N. Maestas. 2009. Does Medicare Save Lives? The Quarterly Journal of Economics 124(2) 597-636.

Carey, K. 2015. Measuring the hospital length of stay/readmission cost trade-off under a bundled payment mechanism. Health Economics 24(7) 790-802.

Carey, Kathleen, Meng-Yun Lin. 2014. Hospital length of stay and readmission an early investigation. Medical Care Research and Review 71(1) 99-111.

Centers for Medicare \& Medicaid Services. $2015 . \quad$ Hospital quality initiative: Hospital compare (accessed 8/14/15). URL https://www.cms.gov/Medicare/ Quality-Initiatives-Patient-Assessment-Instruments/HospitalQualityInits/ HospitalCompare.html.

Centers for Medicare \& Medicaid Services. 2016. Hospital value-based purchasing. URL https:// WWw.cms.gov/Medicare/Quality-Initiatives-Patient-Assessment-Instruments/ hospital-value-based-purchasing/index.html. Accessed March 10, 2016.

Chan, C. W., V. F. Farias, N. Bambos, G. Escobar. 2012. Optimizing icu discharge decisions with patient readmissions. Operations Research 60 1323-1342. 
Clark, C. 2012. Stop using in-hospital mortality rates to judge quality. HealthLeaders Media (accessed 08/14/15). URL http://healthleadersmedia.com/content.cfm?content_id=275021\& page $=1$ \& top $i \mathrm{c}=$ qua.

Clark, J. R., R. S. Huckman. 2012. Broadening focus: Spillovers, complementarities, and specialization in the hospital industry. Management Science 58(4) 708-722.

Dobkin, C. 2003. Hospital staffing and inpatient mortality. working paper .

Elixhauser, A., C. Steiner, D. R. Harris Robert, R. M. Coffey. 1998. Comorbidity measures for use with administrative data. Medical care 36(1) 8-27.

Fleming, C. 2012. Medicare hospital quality reporting brings little or no mortality improvement. Health Affairs Blog (accessed 08/14/15). URL http://healthaffairs.org/blog/2012/03/06/ medicare-hospital-quality-reporting-brings-little-or-no-mortality-improvement/.

Goyal, N., J. R. Zubizarreta, D. S. Small, S. A. Lorch. 2013. Length of stay and readmission among late preterm infants: an instrumental variable approach. Hospital Pediatrics 3 7-15.

Grady, J. N., Z. Lin, Y. Wang, C. Nwosu, M. Keenan, K. Bhat, H. Krumholz, S. Bernheim. 2013. 2013 measures updates and specifications: Acute myocardial infarction, heart failure, and pneumonia 30-day risk-standardized mortality measure (version 7.0). Yale University/Yale-New Haven Hospital-Center for Outcomes Research \& Evaluation (Yale-CORE): Technical Report. Accessed August 8, 2015.

Hamilton, Barton H, Vivian Ho, Dana P Goldman. 2000. Queuing for surgery: is the us or canada worse off? Review of Economics and Statistics 82(2) 297-308.

Ho, V., B. H. Hamilton, L. L. Roos. 2000. Multiple approaches to assessing the effects of delays for hip fracture patients in the United States and Canada. Health Services Research 34 1499-518.

Jaeker, J. B., A. L. Tucker. 2015. Past the point of speeding up: The negative effects of workload saturation on efficiency and quality. Management Science, to appear .

Jha, A. K. 2013. The 30-day readmission rate: Not a quality measure but an accountability measure. accessed 12/01/15. URL https://blogs.sph.harvard.edu/ashish-jha/ the-30-day-readmission-rate-not-a-quality-measure-but-an-accountability-measure/.

Jha, Ashish K., E. John Orav, Zhonghe Li, Arnold M. Epstein. 2007. The inverse relationship between mortality rates and performance in the hospital quality alliance measures. Health Affairs 26(4) 1104-1110.

Joynt, K. E., A. K. Jha. 2012. Thirty-day readmissionstruth and consequences. New England Journal of Medicine 366(15) 1366-1369.

Kaboli, P. J., J.T. Go, J. Hockenberry, J.M. Glasgow, S.R. Johnson, G.E. Rosenthal, M.P. Jones, M. Vaughan-Sarrazin. 2012. Associations between reduced hospital length of stay and 30-day readmission rate and mortality: 14-year experience in 129 veterans affairs hospitals. Annals of internal medicine 157(12) 837-845.

KC, D., B. R. Staats, F. Gino. 2013. Learning from my success and from others' failure: Evidence from minimally invasive cardiac surgery. Management Science 59(11) 2435-2449. 
Kc, D., C. Terwiesch. 2009. Impact of workload on service time and patient safety: An econometric analysis of hospital operations. Management Science 55 1486-1498.

Kc, D., C. Terwiesch. 2012. An econometric analysis of patient flows in the cardiac intensive care unit. Manufacturing \& Service Operations Management 14(1) 50-65.

Kuntz, L., R. Mennicken, S. Scholtes. 2015. Stress on the ward: Evidence of safety tipping points in hospitals. Management Science 61(4) 754-771.

Makowsky, M. D., E. Y. Klein. 2015. Identifying the relationship between length of hospital stay and the probability of readmission. working paper .

Murphy, K. M., R. H. Topel. 2006. The value of health and longevity. Journal of Political Economy 114 871-904.

National Quality Forum. 2009. Measure Submission and Evaluation Worksheet 5.0: Pulmonary Project: Hospital 30day, All-cause, Risk-standardized Mortality Rate (RSMR) following Pneumonia Hospitalization. Tech. Rep. 0468 .

Nichols, G.A., K. Reynolds, M.G. Butler, T.M. Kimes, W.W. Chan. 2014. Association of acute heart failure length of stay with subsequent re-hospitalization and all-cause mortality among patients with preserved vs. reduced ejection fraction. Circulation 130 A11307.

Powell, A., S. Savin, N. Savva. 2012. Physician workload and hospital reimbursement: Overworked physicians generate less revenue per patient. Manufacturing \& Service Operations Management 14(4) 512-528.

Press, M. J., D. P. Scanlon, A. M. Ryan, J. Zhu, A. S. Navathe, J. N. Mittler, K. G. Volpp. 2013. Limits of readmission rates in measuring hospital quality suggest the need for added metrics. Health Affairs 32(6) 1083-1091.

Reynolds, K., M. G. Butler, T. M. Kimes, A. G. Rosales, W. Chan, G. A. Nichols. 2015. Relation of acute heart failure hospital length of stay to subsequent readmission and all-cause mortality. The American Journal of Cardiology 116(3) $400-405$.

Rinne, S. T., B. F. Collins, M. Perkins, D. H. Au, C.-F. Liu. 2014. Weekend discharges after copd hospitalizations are not associated with a higher risk of readmission. American Journal of Respiratory and Critical Care Medicine 189(American Thoracic Society 2014 International Conference, Meeting Abstracts) A3717.

Ryan, Andrew M., James F. Burgess, Christopher P. Tompkins, Stanley S. Wallack. 2009. The relationship between medicare's process of care quality measures and mortality. INQUIRY: The Journal of Health Care Organization, Provision, and Financing 46(3) 274-290.

Ryan, J. W., E. D. Peterson, A. Y. Chen, M.T. Roe, E. M. Ohman, C. P. Cannon, P. B. Berger, J. F. Saucedo, E. R. DeLong, S. L. Normand, C. V. Pollack Jr, D. J. Cohen. 2005. Optimal timing of intervention in non-ST-segment elevation acute coronary syndromes: insights from the CRUSADE (Can Rapid risk stratification of Unstable angina patients Suppress ADverse outcomes with Early implementation of the ACC/AHA guidelines) Registry. Circulation 112 3049-3057.

Taheri, P. A., D. A. Butz, L. J. Greenfield. 2000. Length of stay has minimal impact on the cost of hospital admission. Journal of the American College of Surgeons 191(2) 123-130. 
The Henry J. Kaiser Family Foundation. 2014. Hospital adjusted expenses per inpatient day. URL http: / kff . org/other/state-indicator/expenses-per-inpatient-day/. Accessed July 3, 2014.

Varnava, A. M., J. E. Sedgwick, A. Deaner, K. Ranjadayalan, A. D. Timmis. 2002. Restricted weekend service inappropriately delays discharge after acute myocardial infarction. Heart 87 216-219.

Williams, T. A., K.M. Ho, G.J. Dobb, J.C. Finn, M. Knuiman, S.A.R. Webb. 2010. Effect of length of stay in intensive care unit on hospital and long-term mortality of critically ill adult patients. British journal of anaesthesia 104(4) 459-464.

Wong, H., R. C. Wu, G. Tomlinson, M. Caesar, H. Abrams, M. W. Carter, D. Morra. 2009. How much do operational processes affect hospital inpatient discharge rates? Journal of Public Health 31 546-553.

Wooldridge, J. M. 2010. Econometric analysis of cross section and panel data. MIT press. 


\section{Appendix}

Table A.1 AMI Patients Data Selection

\begin{tabular}{|c|c|c|c|}
\hline Sample & Observations & $\%$ prior & $\%$ initial \\
\hline All Admissions in 2000-2011, except for Nov-Dec 2011 admits/discharges & 185071967 & NA & 100.0 \\
\hline Excluding overlapping admissions & 158571615 & 85.7 & 85.7 \\
\hline Excluding post-acute care & 132154789 & 83.3 & 71.4 \\
\hline Excluding stays with hospital transfers & 117858724 & 89.2 & 63.7 \\
\hline Excluding those in facilities not paid under PPS & 112157751 & 95.2 & 60.6 \\
\hline Excluding stays that are not FFS & 102793279 & 91.7 & 55.5 \\
\hline Excluding non-AMI patients & 2443336 & 2.4 & 1.3 \\
\hline Excluding those admitted within 30 days of prior admission's discharge & 2351439 & 96.2 & 1.3 \\
\hline Excluding hospitals with less than 25 visits & 2343377 & 99.7 & 1.3 \\
\hline Excluding patients with inpatient service related discharge destinations & 2017891 & 86.1 & 1.1 \\
\hline Excluding non-elderly admissions & 1794821 & 88.9 & 1.0 \\
\hline Excluding those that died during the & 1789470 & 99.7 & 1.0 \\
\hline Excluding those that left against medical advice & 1781363 & 99.5 & 1.0 \\
\hline Excluding those with unknown race or not residing in the US & 1775221 & 99.7 & 1.0 \\
\hline Excluding elective patients (including unknown elective status) & 1669264 & 94.0 & 0.9 \\
\hline Excluding same day discharge (for AMI patients only) & 1669258 & 100.0 & 0.9 \\
\hline Excluding cost outliers & 1615888 & 96.8 & 0.9 \\
\hline Excluding length of stays beyond the 99 th percentile (19 days) & 1600420 & 99.0 & 0.9 \\
\hline
\end{tabular}

Table A.2 HF Patients Data Selection

\begin{tabular}{|c|c|c|c|}
\hline Sample & Observations & $\%$ prior & $\%$ initial \\
\hline All Admissions in 2000-2011, except for Nov-Dec 2011 admits/discharges & 185071967 & NA & 100.0 \\
\hline Excluding overlapping admissions & 158571615 & 85.7 & 85.7 \\
\hline Excluding post-acute care & 132154789 & 83.3 & 71.4 \\
\hline Excluding stays with hospital transfers & 117858724 & 89.2 & 63.7 \\
\hline Excluding those in facilities not paid under PPS & 112157751 & 95.2 & 60.6 \\
\hline Excluding stays that are not FFS & 102793279 & 91.7 & 55.5 \\
\hline Excluding non-HF patients & 6197102 & 6.0 & 3.3 \\
\hline Excluding those admitted within 30 days of prior admission's discharge & 5642096 & 91.0 & 3.0 \\
\hline Excluding hospitals with less than 25 visits & 5637145 & 99.9 & 3.0 \\
\hline Excluding patients with inpatient service related discharge destinations & 5273331 & 93.5 & 2.8 \\
\hline Excluding non-elderly admissions & 4641460 & 88.0 & 2.5 \\
\hline Excluding those that died during the stay & 4633477 & 99.8 & 2.5 \\
\hline Excluding those that left against medical advice & 4616545 & 99.6 & 2.5 \\
\hline Excluding those with unknown race or not residing in the US & 4602498 & 99.7 & 2.5 \\
\hline Excluding elective patients (including unknown elective status) & 4134583 & 89.8 & 2.2 \\
\hline Excluding cost outliers & 4086775 & 98.8 & 2.2 \\
\hline Excluding length of stays beyond the 99th percentile (18 days) & 4047914 & 99.0 & 2.2 \\
\hline
\end{tabular}


Table A.3 PNE Patients Data Selection

\begin{tabular}{|c|c|c|c|}
\hline Sample & Observations & $\%$ prior & $\%$ initial \\
\hline All Admissions in 2000-2011, except for Nov-Dec 2011 admits/discharges & 185071967 & NA & 100.0 \\
\hline Excluding overlapping admissions & 158571615 & 85.7 & 85.7 \\
\hline Excluding post-acute care & 132154789 & 83.3 & 71.4 \\
\hline Excluding stays with hospital transfers & 117858724 & 89.2 & 63.7 \\
\hline Excluding those in facilities not paid under PPS & 112157751 & 95.2 & 60.6 \\
\hline Excluding stays that are not FFS & 102793279 & 91.7 & 55.5 \\
\hline Excluding non-PNE patients & 5467468 & 5.3 & 3.0 \\
\hline Excluding those admitted within 30 days of prior admission's discharge & 5266804 & 96.3 & 2.8 \\
\hline Excluding hospitals with less than 25 visits & 5262672 & 99.9 & 2.8 \\
\hline Excluding patients with inpatient service related discharge destinations & 4776512 & 90.8 & 2.6 \\
\hline Excluding non-elderly admissions & 4131502 & 86.5 & 2.2 \\
\hline Excluding those that died during the stay & 4122426 & 99.8 & 2.2 \\
\hline Excluding those that left against medical advice & 4111520 & 99.7 & 2.2 \\
\hline Excluding those with unknown race or not residing in the US & 4098850 & 99.7 & 2.2 \\
\hline Excluding elective patients (including unknown elective status) & 3761247 & 91.8 & 2.0 \\
\hline Excluding cost outliers & 3723678 & 99.0 & 2.0 \\
\hline Excluding length of stays beyond the 99th percentile (19 days) & 3686544 & 99.0 & 2.0 \\
\hline
\end{tabular}



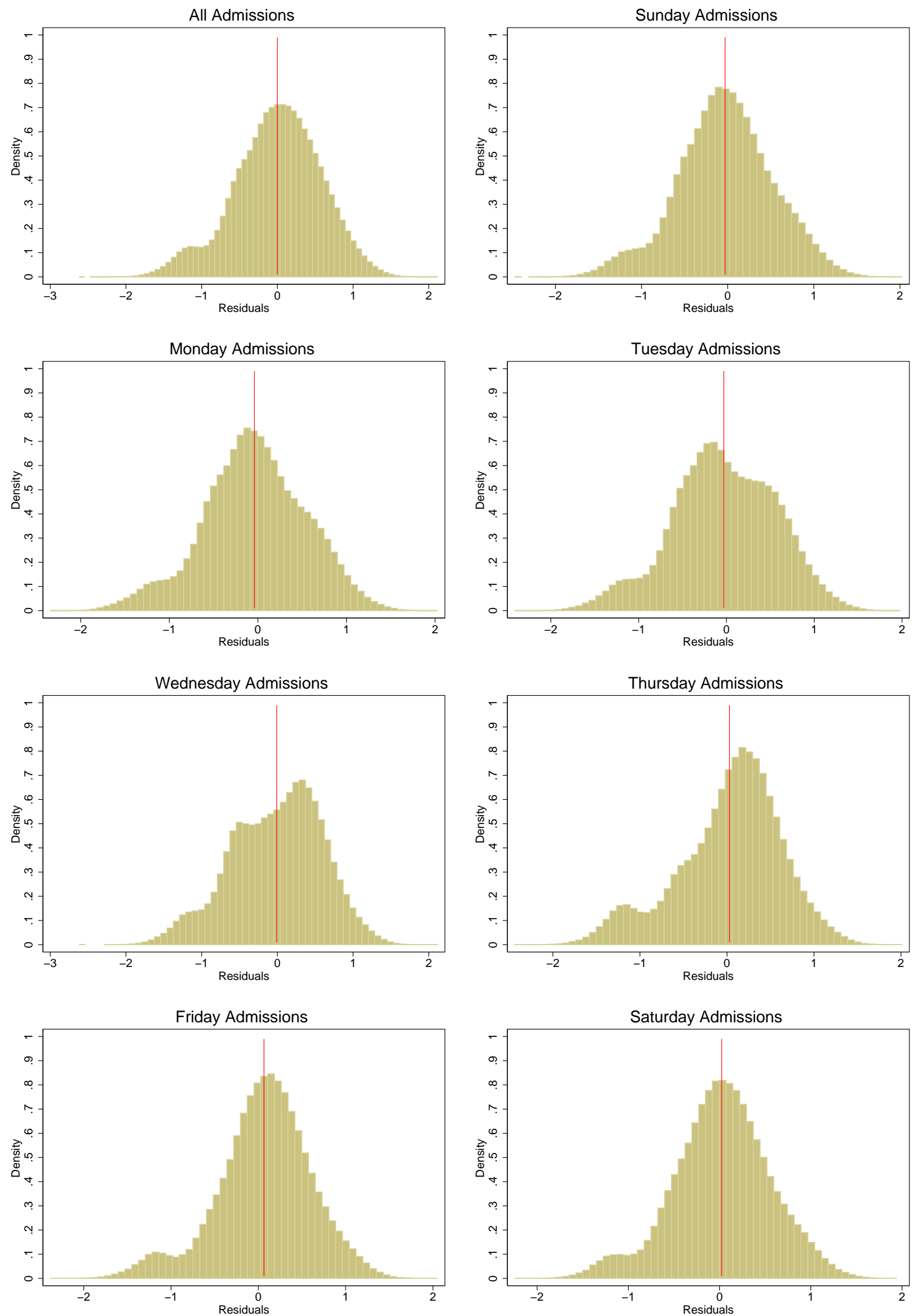

Figure A.1 Residual histograms of $\log (L O S)$ regression for HF patients by admission day-of-week 
Table A.4 Robustness Check of Mortality in 3 days Model Results for AMI Patients

\begin{tabular}{l|ccccc}
\hline & Non-profit & Big & $\begin{array}{c}\text { w/o Surgical } \\
\text { Procedures }\end{array}$ & $\begin{array}{c}\text { w/ Weekend } \\
\text { Admits }\end{array}$ & $\begin{array}{c}\text { Random } \\
\text { Episode }\end{array}$ \\
\hline $\log (\mathrm{LOS})$ & -0.16 & $-0.21^{*}$ & $-0.37 * *$ & -0.14 & $-0.22^{+}$ \\
& $(0.12)$ & $(0.11)$ & $(0.14)$ & $(0.09)$ & $(0.11)$ \\
\hline IV & $-0.06 * * *$ & $-0.06 * * *$ & $-0.07 * * *$ & $-0.06 * * *$ & $-0.06 * * *$ \\
& $(0.01)$ & $(0.01)$ & $(0.00)$ & $(0.00)$ & $(0.00)$ \\
\hline Num. of obs. & 809756 & 919653 & 297244 & 1564518 & 1026380 \\
Wald $\chi^{2}$ test & 1.14 & 2.69 & 2.25 & 1.03 & 2.43 \\
Wald p-value & 0.29 & 0.10 & 0.13 & 0.31 & 0.12 \\
\hline
\end{tabular}

Table A.5 Robustness Check of Mortality in 7 days Model Results for AMI Patients

\begin{tabular}{l|ccccc}
\hline & Non-profit & Big & $\begin{array}{c}\text { w/o Surgical } \\
\text { Procedures }\end{array}$ & $\begin{array}{c}\text { w/ Weekend } \\
\text { Admits }\end{array}$ & $\begin{array}{c}\text { Random } \\
\text { Episode }\end{array}$ \\
\hline $\log (\mathrm{LOS})$ & -0.13 & $-0.20^{+}$ & -0.21 & -0.10 & $-0.20^{*}$ \\
& $(0.11)$ & $(0.11)$ & $(0.13)$ & $(0.08)$ & $(0.10)$ \\
\hline IV & $-0.06^{* * *}$ & $-0.06^{* * *} *$ & $-0.07 * * *$ & $-0.06^{* * *}$ & $-0.06^{* * *}$ \\
& $(0.01)$ & $(0.01)$ & $(0.00)$ & $(0.00)$ & $(0.00)$ \\
\hline Num. of obs. & 822058 & 922794 & 320390 & 1587332 & 1051758 \\
Wald $\chi^{2}$ test & 2.22 & 4.37 & 0.78 & 2.17 & 5.01 \\
Wald p-value & 0.14 & 0.04 & 0.38 & 0.14 & 0.03 \\
\hline Note. Robust standard errors in parentheses. ${ }^{+}(p<0.1), *(p<0.05), * *(p<0.01), * * *(p<$ \\
0.001).
\end{tabular}

Table A.6 Robustness Check of Mortality in 60 days Model Results for AMI Patients

\begin{tabular}{l|ccccc}
\hline & Non-profit & Big & $\begin{array}{c}\text { w/o Surgical } \\
\text { Procedures }\end{array}$ & $\begin{array}{c}\text { w/ Weekend } \\
\text { Admits }\end{array}$ & $\begin{array}{c}\text { Random } \\
\text { Episode }\end{array}$ \\
\hline $\log (\mathrm{LOS})$ & -0.03 & $-0.10^{+}$ & -0.12 & -0.04 & $-0.13^{*}$ \\
& $(0.07)$ & $(0.06)$ & $(0.08)$ & $(0.05)$ & $(0.06)$ \\
\hline IV & $-0.06^{* * *}$ & $-0.06^{*} * *$ & $-0.07 * * *$ & $-0.06 * * *$ & $-0.06 * * *$ \\
& $(0.01)$ & $(0.01)$ & $(0.00)$ & $(0.00)$ & $(0.00)$ \\
\hline Num. of obs. & 826128 & 924268 & 335021 & 1599681 & 1065412 \\
Wald $\chi^{2}$ test & 11.34 & 31.73 & 8.78 & 24.72 & 30.27 \\
Wald p-value & 0.00 & 0.00 & 0.01 & 0.00 & 0.00 \\
\hline Note. Robust standard errors in parentheses. ${ }^{+}(p<0.1), *(p<0.05), * *(p<0.01), * * *(p<$ \\
0.001).
\end{tabular}


Table A.7 Robustness Check of Mortality in 3 days Model Results for PNE Patients

\begin{tabular}{l|ccccc}
\hline & Non-profit $\dagger$ & Big & $\begin{array}{c}\text { w/o Surgical } \\
\text { Procedures } \dagger\end{array}$ & $\begin{array}{c}\text { w/ Weekend } \\
\text { Admits } \dagger\end{array}$ & $\begin{array}{c}\text { Random } \\
\text { Episode }\end{array}$ \\
\hline $\log ($ LOS $)$ & $-0.34^{*}$ & $-0.25^{* *}$ & $-0.42^{* *}$ & $-0.39 * * *$ & $-0.24 *$ \\
& $(0.14)$ & $(0.09)$ & $(0.15)$ & $(0.10)$ & $(0.12)$ \\
\hline IV & $-0.05^{* * *}$ & $-0.05^{* * *}$ & $-0.05^{* * *}$ & $-0.04 * * *$ & $-0.05 * * *$ \\
& $(0.00)$ & $(0.00)$ & $(0.00)$ & $(0.00)$ & $(0.00)$ \\
\hline Num. of obs. & 1409390 & 1368968 & 1504662 & 3089047 & 1772254 \\
Wald $\chi^{2}$ test & 8.67 & 10.74 & 11.20 & 19.79 & 6.35 \\
Wald p-value & 0.00 & 0.00 & 0.00 & 0.00 & 0.01 \\
\hline Note. Robust standard errors in parentheses. ${ }^{+}(p<0.1), *(p<0.05), * *(p<0.01), * * *(p<$ \\
$0.001) . \dagger$ Estimated using two-step estimator.
\end{tabular}

Table A.8 Robustness Check of Mortality in 7 days Model Results for PNE Patients

\begin{tabular}{l|ccccc}
\hline & Non-profit & Big $\dagger$ & $\begin{array}{c}\text { w/o Surgical } \\
\text { Procedures } \dagger\end{array}$ & $\begin{array}{c}\text { w/ Weekend } \\
\text { Admits }\end{array}$ & $\begin{array}{c}\text { Random } \\
\text { Episode } \dagger\end{array}$ \\
\hline $\log (\mathrm{LOS})$ & $-0.38^{* * *}$ & $-0.29^{* *}$ & $-0.46^{* * *}$ & $-0.45^{* * *}$ & $-0.31^{* *}$ \\
& $(0.09)$ & $(0.11)$ & $(0.11)$ & $(0.09)$ & $(0.10)$ \\
\hline $\mathrm{IV}$ & $-0.05^{* * *}$ & $-0.05 * * *$ & $-0.05^{* * *}$ & $-0.04 * * *$ & $-0.05 * * *$ \\
& $(0.00)$ & $(0.00)$ & $(0.00)$ & $(0.00)$ & $(0.00)$ \\
\hline Num. of obs. & 1418969 & 1370499 & 1557358 & 1418969 & 1810769 \\
Wald $\chi^{2}$ test & 28.98 & 13.51 & 27.07 & 28.98 & 19.46 \\
Wald p-value & 0.00 & 0.00 & 0.00 & 0.00 & 0.00 \\
\hline Note. Robust standard errors in parentheses. ${ }^{+}(p<0.1), *(p<0.05), * *(p<0.01), * * *(p<$ \\
$0.001)$. Estimated using two-step estimator.
\end{tabular}

Table A.9 Robustness Check of Mortality in 60 days Model Results for PNE Patients

\begin{tabular}{l|ccccc}
\hline & Non-profit & Big & $\begin{array}{c}\text { w/o Surgical } \\
\text { Procedures }\end{array}$ & $\begin{array}{c}\text { w/ Weekend } \\
\text { Admits }\end{array}$ & $\begin{array}{c}\text { Random } \\
\text { Episode }\end{array}$ \\
\hline $\log (\mathrm{LOS})$ & -0.08 & $-0.10^{*}$ & $-0.14^{*}$ & $-0.15^{* * *}$ & $-0.10^{+}$ \\
& $(0.05)$ & $(0.05)$ & $(0.06)$ & $(0.04)$ & $(0.05)$ \\
\hline IV & $-0.05^{* * *}$ & $-0.05^{* * *}$ & $-0.05^{* * *}$ & $-0.04 * * *$ & $-0.05^{* * *}$ \\
& $(0.00)$ & $(0.00)$ & $(0.00)$ & $(0.00)$ & $(0.00)$ \\
\hline Num. of obs. & 1421766 & 1371079 & 1584598 & 3141480 & 1831370 \\
Wald $\chi^{2}$ test & 43.39 & 51.47 & 37.14 & 83.91 & 41.80 \\
Wald p-value & 0.00 & 0.00 & 0.00 & 0.00 & 0.00 \\
\hline
\end{tabular}

Note. Robust standard errors in parentheses. ${ }^{+}(p<0.1), *(p<0.05), * *(p<0.01), * * *(p<$ $0.001)$. 